\title{
Chemical Profiling, Antioxidant, Anticholinesterase, and Antiprotozoal Potentials of Artemisia copa Phil. (Asteraceae)
}

María José Larrazábal-Fuentes ${ }^{1 \dagger}$, Carlos Fernández-Galleguillos ${ }^{2 \dagger}$, Jenifer Palma-Ramírez ${ }^{1}$, Javier Romero-Parra ${ }^{3}$, Kevin Sepúlveda ${ }^{4}$, Alexandra Galetovic ${ }^{5}$, Jorge González ${ }^{4}$, Adrián Paredes ${ }^{6}$, Jorge Bórquez ${ }^{6}$, Mario J. Simirgiotis ${ }^{2 \star}$ and Javier Echeverría ${ }^{7 *}$

\section{OPEN ACCESS}

Edited by:

Michał Tomczyk

Medical University of Bialystok, Poland

Reviewed by:

Sengul Uysal,

Selçuk University, Turkey

Daniil Nikolaevich Olennikov,

Institute of General and Experimental

Biology (RAS), Russia

Susana Gorzalczany,

University of Buenos Aires, Argentina

*Correspondence:

Mario J. Simirgiotis mario.simirgiotis@uach.cl

Javier Echeverría

javier.echeverriam@usach.c

${ }^{\dagger}$ These authors share first authorship

Specialty section:

This article was submitted to

Ethnopharmacology,

a section of the journal

Frontiers in Pharmacology

Received: 12 August 2020

Accepted: 22 October 2020

Published: 04 December 2020

Citation:

Larrazábal-Fuentes MJ, FernándezGalleguillos C, Palma-Ramírez J,

Romero-Parra J, Sepúlveda K, Galetovic A, González J, Paredes A,

Bórquez J, Simirgiotis MJ and Echeverría J (2020) Chemical Profiling, Antioxidant, Anticholinesterase, and Antiprotozoal Potentials of Artemisia copa Phil. (Asteraceae).

Front. Pharmacol. 11:594174. doi: 10.3389/fphar.2020.594174
${ }^{1}$ Unidad Alimentos, Departamento de Ciencias de los Alimentos y Nutrición, Facultad de Ciencias de la Salud, Universidad de Antofagasta, Antofagasta, Chile, ${ }^{2}$ Instituto de Farmacia, Facultad de Ciencias, Universidad Austral de Chile, Valdivia, Chile,

${ }^{3}$ Departamento de Química Orgánica y Fisicoquímica, Facultad de Ciencias Químicas y Farmacéuticas, Universidad de Chile, Santiago, Chile, ${ }^{4}$ Unidad de Parasitología Molecular, Departamento de Tecnología Médica, Facultad de Ciencias de la Salud, Universidad de Antofagasta, Antofagasta, Chile, ${ }^{5}$ Departamento Biomédico, Universidad de Antofagasta, Antofagasta, Chile, ${ }^{6}$ Laboratorio de Productos Naturales, Departamento de Química, Facultad de Ciencias Básicas, Universidad de Antofagasta, Antofagasta, Chile, ${ }^{7}$ Departamento de Ciencias del Ambiente, Facultad de Química y Biología, Universidad de Santiago de Chile, Santiago, Chile

Artemisia copa Phil. (Asteraceae) (known as copa-copa) is a native species of Chile used as an infusion in traditional medicine by Atacameños people in the Altiplano, highlands of northern Chile. In this research, we have investigated for the first time the cholinesterase inhibition potential against acetylcholinesterase (AChE) and butyrylcholinesterase (BChE), and the chemical profiling of the infusions prepared from the aerial parts of $A$. copa by high resolution spectrometry. In addition, total phenolic, total flavonoid content, antioxidant (DPPH, FRAP, and ORAC) and antiprozoal activity were tested. Artemisia copa showed good inhibitory activity against AChE and BChE $(3.92 \pm 0.08 \mu \mathrm{g} / \mathrm{ml}$ and $44.13 \pm 0.10 \mu \mathrm{g} /$ $\mathrm{ml})$. The infusion displayed a total phenolics content of $155.6 \pm 2.9 \mathrm{mg}$ of gallic acid equivalents $/ \mathrm{g}$ and total flavonoid content of $5.5 \pm 0.2 \mathrm{mg}$ quercetin equivalents $/ \mathrm{g}$. Additionally, trypanocidal activity against Trypanosoma cruzi was found ( $L D_{50}$ of $131.8 \mu \mathrm{g} / \mathrm{ml})$. Forty-seven metabolites were detected in the infusion of $A$. copa including several phenolic acids and flavonoids which were rapidly identified using ultrahigh performance liquid chromatography orbitrap mass spectrometry analysis (UHPLC-Orbitrap-MS) for chemical profiling. The major compounds identified in the infusions were studied by molecular docking against AChE and BChE. The UHPLCMS fingerprints generated can be also used for the authentication of these endemic

\footnotetext{
Abbreviations: AAPH, 2,2'-azobis(2-amidinopropane) dihydrochloride; AChE, acetylcholinesterase; ANOVA, one-way analysis of variance; ATCI, acetyl-thiocholine iodide; BChE, butyrylcholinesterase; ChE, cholinesterase; DPPH, 2,2,1-diphenyl1-picrylhydrazyl; DTNB, 5-dithio-bis(2-nitrobenzoic) acid; FRAP, ferric reducing antioxidant power; GAE, gallic acid equivalent; IC50, half maximal inhibitory concentration; MS, mass spectrometry; NO, nitric oxide; ORAC, oxygen radical absorbance capacity; OT, orbitrap technology; PDA, photodiode array detection; PTFE, polytetrafluoroethylene; QE, quercetin equivalent; ROS, reactive oxygen species; RP, reverse phase; TE, trolox equivalents; TEAC, trolox equivalent antioxidant capacity; TFC, total flavonoid content; TIC, total ion chromatogram; TPC, total phenolic content; TPTZ, 2,4,6-tri(2-pyridyl)-striazine; Trolox, 6-hydroxy-2,5,7,8-tetramethylchromane-2-carboxylic acid; UV, ultraviolet; UHPLC, ultra high performance chromatography; HESI II, electrospray heated ionization probe.
} 
species. These findings reveal that $A$. copa infusions can be used as beverages with protective effects.

Keywords: Artemisia copa, Asteraceae, traditional medicine, HPLC-MS, anti-Trypanosoma cruzi, Anti-Trypanosoma activity, cholinesterase inhibition

\section{INTRODUCTION}

The Andean Altiplano is a South American high plateau located at about 3,700 $\mathrm{m}$ above sea level shared by Bolivia, Argentina, part of southern Peru, and Chile. These locations have ecological and environmental importance, and several ancient civilizations arose there, which uses the special fauna and flora as food and medicines for centuries. Artemisia copa Phil. (Asteraceae) also knows as "copa-copa" is a Chilean altiplano plant characterized by having small green leaves (Figure 1) and used as an infusion in popular medicine (Giberti, 1983) in the Atacama Desert, North West of Argentina and Altiplano in Northern Chile. It grows between 3,500 and 4,250 $\mathrm{m}$ above sea level.

The A. copa infusion is used in traditional medicine for the treatment of colds, pneumonia, hypertension, pain, for gall bladder problems, as digestive, often combined with sodium bicarbonate. The infusion mixed with milk is used for stomach pain, cold, and colic. In the form of baths or incense, it serves to combat "mal de aire" and toothache. As a bath, it serves for bone pain. People attribute sedative properties to the species the ability to cause vivid dreams (Giberti, 1983; Montes and Wilkomirsky, 1985; De Nucci, 1988; Paniagua-Zambrana et al., 2020).

Different compounds have been identified in the extracts of aerial parts of Artemisia copa. In the dichloromethane and ethanolic extracts have been isolated the flavonoids spinacetin, jaceosidin, axillarin, penduletin, tricin, chrysoeriol (Moscatelli et al., 2006), chrysosplenetin (Morales et al., 2003), jaceidin (de la Fuente et al., 1986), 7-methyljaceidin, kaempherol-6-methyleter3-O-rhamnoglucosyde, and luteolin (Catalán et al., 2007). The guaianolides achillin and deacetylmatricarin have been isolated (de la Fuente et al., 1986). The coumarin scopoletin and phenolic acid $p$-coumaric acid have also been identified (Moscatelli et al., 2006). The essential oils of leaves, twigs, and flowers have been identified monoterpenoids as chrysanthenone, chrysanthenyl acetate, $\beta$-thujone, $\gamma$-terpinene, cis-2-menthenol, limonene and $\alpha$-pinene, sesquiterpenoids as chamazulene, linalool and linalyl acetate, and homoterpene and other miscellaneous compounds as butyric acid (Catalan et al., 1982; Arze et al., 2004; Catalán et al., 2007; Larrazabal-Fuentes et al., 2019).

A. copa has been shown to possess significant biological activities such as antioxidant (Pérez-García et al., 2001; Catalán et al., 2007), analgesic and topical anti-inflammatory activities via inhibition of nitric oxide production, reduction of prostaglandin E2 levels and inhibition of cyclooxygenase-2 activity (Miño et al., 2004), inhibition of synovial phospholipase A2 activity (Moscatelli et al., 2006), spasmolytic activity (Gorzalczany et al., 2013a) and vasorelaxant and hypotensive effects through the inhibition of $\mathrm{Ca}^{2+}$ influx via membranous calcium channels and intracellular stores (Gorzalczany et al., 2013b).

Numerous results are reporting on the anti-trypanosomal activity the genus Artemisia, artemisinin, and its derivatives and other phytochemicals from Artemisia species (Sen et al., 2007; Aloui et al., 2016). However, more intensive research is required to explore the full potential of diverse Artemisia species and their chemical ingredients for the eradication of trypanosomal infections (Naß and Efferth, 2018).

Until now, no studies have been reported on the antiprotozoal and enzyme inhibitory properties against cholinesterase of $A$.

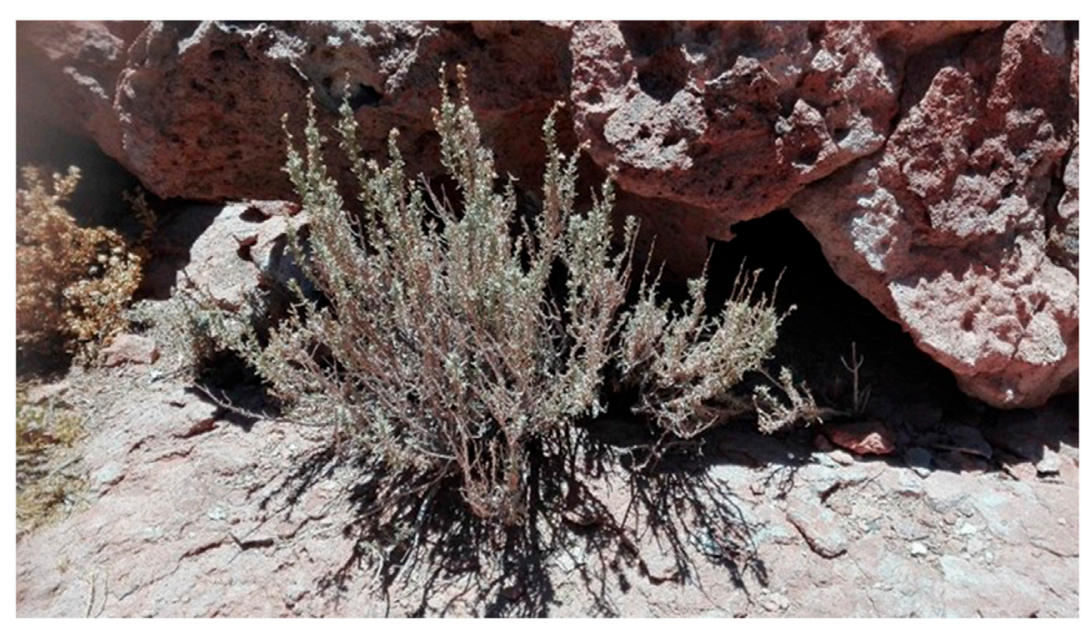

FIGURE 1 | Artemisia copa Phil (Asteraceae). 
copa. In the same way, no metabolomic analyzes of the chemical compounds present in A. copa have been reported. Ultra-highperformance liquid chromatography (UHPLC) coupled with high-resolution mass spectrometry (HR-MS) is a modern and fast technique that allows the identification of accurate mass analysis. Moreover, is today a key tool for plant chemotaxonomy or metabolic profiling of plant samples (Simirgiotis et al., 2016b; Echiburu-Chau et al., 2017; Areche et al., 2020; Rodriguez et al., 2020).

Since herbal native infusions are receiving increasing attention for the number of physiological benefits they can bring to human health (Coelho et al., 2016) and as part of our continuing research of medicinal plants from Atacama Desert (Echiburu-Chau et al., 2017; Ardiles et al., 2018; Areche et al., 2020; Rodriguez et al., 2020) and native plants with potentiality as cholinesterase inhibitors (Barrientos et al., 2020a; Barrientos et al., 2020b), we investigated the infusions of the medicinal plant $A$. copa using screening tests to evaluate their antioxidant, antiprotozoal and enzyme inhibitory properties against cholinesterase and high-resolution UHPLC-MS/MS for the chemical profiling for the first time. Additionally, molecular docking of the major compounds was investigated.

\section{MATERIALS AND METHODS}

\section{Chemicals and Regents}

Solvents type UHPLC-MS, formic acid type LC-MS, and chloroform were from Merck (Santiago, Chile). Ultrapure water was obtained from a water purification system brand Millipore (Milli-Q Merck Millipore, Chile). Flavonol standards, (catechin, isoflavones, and flavonoids, all standards with a high purity: $95 \%$ by HPLC) were acquired from ChromaDex (Santa Ana, CA, United States), Sigma Aldrich (Saint Louis, Mo, United States), or Extrasynthèse (Genay, France). Folin-Ciocalteu reagent, $\mathrm{NaOH}$, $\mathrm{Na}_{2} \mathrm{CO}_{3}, \mathrm{AlCl}_{3}, \mathrm{FeCl}_{3}, \mathrm{HCl}, \mathrm{NaNO}_{2}$, trichloroacetic acid, quercetin, 6-hydroxy-2,5,7,8-tetramethylchromane-2-carboxylic acid (Trolox), sodium acetate, Gallic acid, TPTZ, nitroblue tetrazolium, DPPH (1,1-diphenyl-2-picrylhydrazyl radical), acetylcholinesterase (AChE), butyrylcholinesterase (BChE), 2,2'Azobis (2-amidinopropane) dihydrochloride (AAPH), were acquired from Sigma-Aldrich Chemical Company (Santiago, Chile).

\section{Plant Material}

Artemisia copa Phil. (Asteraceae) was collected in El Tatio, Antofagasta, Región de Antofagasta, Chile in November 2016. Voucher specimens for the herbarium are kept at the Laboratory of Natural Products of the Universidad de Antofagasta under the number: AC20161115.

\section{Preparation of the Infusion of $\boldsymbol{A}$. copa}

The infusion was prepared using $3 \mathrm{~g}$ of dried milled aerial parts (stems and leaves) with $250 \mathrm{ml}$ of boiling deionized water for $30 \mathrm{~min}$. The infusion was filtered (Whatman 1), lyophilized (Labconco $2.5 \mathrm{~L}$ ), and refrigerated until use at $4^{\circ} \mathrm{C}$ (the yield of the aqueous extraction was $8.6 \%$ ).

\section{Ultra-High-Performance Liquid Chromatography-Photodiode Array Detection-Orbitrap Technology-Mass Spectrometry Instrument}

A UHPLC high-resolution MS Dionex Thermo Scientific Ultimate 3000 system connected with a Thermo Q-Exactive focus machine was used. For the analysis, $2 \mathrm{mg}$ per $\mathrm{ml}$ of lyophilized material was dissolved in methanol-distilled water $(1: 1 \mathrm{v} / \mathrm{v})$ and $10 \mu \mathrm{l}$ of filtered solution (PTFE filter) was injected in the instrument, with the specifications set as already informed (Simirgiotis et al., 2016a).

\section{LC Parameters and Mass Spectrometry Parameters}

Liquid chromatography was run using an Acclaim UHPLC C18 column $(150 \times 4.6 \mathrm{~mm}$ ID, $2.5 \mu \mathrm{m}$, Thermo Fisher Scientific, Bremen, Germany) set at $25^{\circ} \mathrm{C}$. The wavelength detection was $354,254,280$, and $330 \mathrm{~nm}$, and PDA was acquired from 200 to $800 \mathrm{~nm}$ for full characterization of peaks. Mobile phases employed were acetonitrile (B) and $1 \%$ formic aqueous solution (A) while the gradient program was: (time in minutes and $\% \mathrm{~B}),(0.00 \mathrm{~min}, 5 \% \mathrm{~B}) ;(5.00 \mathrm{~min}, 5 \% \mathrm{~B})$; (10.00 $\mathrm{min}, 30 \% \mathrm{~B})$; (15.00 $\mathrm{min}, 30 \% \mathrm{~B})$; (20.00 $\mathrm{min}, 70 \% \mathrm{~B})$; (25.00 $\mathrm{min}, 70 \% \mathrm{~B})$; (35.00 $\mathrm{min}, 5 \% \mathrm{~B})$ and $12 \mathrm{~min}$ for column equilibration before injections. The flow rate employed was $1.00 \mathrm{ml} \mathrm{min}{ }^{-1}$, and the injection volume was $10 \mu \mathrm{l}$. Standards dissolved in methanol and infusions were maintained at $10^{\circ} \mathrm{C}$ during storage in the auto-sampler. The HESI II and OT spectrometer parameters were set as informed previously (Simirgiotis et al., 2016c).

\section{HPLC-Photodiode Array Detection Quantitation of Main Phenolic Compounds} in A. copa

Some phenolic compounds were quantified using the UHPLC Dionex $3000 \mathrm{RS}$ with photodiode array detector at flavonoid (255 and $265 \mathrm{~nm})$ and phenolic acid $(330 \mathrm{~nm})$ wavelength as reported previously carried out according to ICH Guideline Q2 (R1) (Barrientos et al., 2020b). Some curves for representative compounds, each one covering six points from 0.01 to $0.5 \mathrm{mg} / \mathrm{ml}$ solutions, in triplicate, and injecting $10 \mathrm{ml}$. The linearity of the chromatographic method developed was confirmed by the coefficients of determination all around 0.999. For kaempferol based compounds the calibration curve was performed with kaempferol standard, $\left(R^{2}=0.9997\right)$ and for quercetin-based compounds, the curve was performed with quercetin standard $\left(R^{2}=0.9989\right)$, and other phenolic acid were quantified using a chlorogenic acid curve $\left(R^{2}=0.9994\right)$. Using PDA, the lowest detection (LOD) and quantification (LOQ) limits, 0.0115 and $0.0322 \mathrm{mg} / \mathrm{L}$ respectively, were obtained for kaempferol, while the highest limits, 0.5543 , and $1.476 \mathrm{mg} / \mathrm{l}$ were for chlorogenic acid. For the quantification the prepared infusion was injected $(10 \mathrm{ml})$ in triplicate at $25^{\circ} \mathrm{C}$. 


\section{Determination of Total Phenolic and Flavonoid Contents}

The analyses of total phenolic compounds and flavonoid content were performed following a previous methodology of FolinCiocalteu (Morales and Paredes, 2014) and the aluminum chloride method (Simirgiotis et al., 2013) in microplate with some modifications. For total phenolic, a standard solution of gallic acid serial dilution $(25-300 \mu \mathrm{g} / \mathrm{ml}$ in $10 \% \mathrm{EtOH})$ was used to prepare a calibration curve. Results were expressed as gallic acid equivalent (GAE)/g of dry plant. Flavonoid content was calculated using a quercetin standard calibration curve $(2.5-250 \mu \mathrm{g} / \mathrm{ml}$ in ethanol) and the results were expressed as mg quercetin equivalents $(\mathrm{QE}) / \mathrm{g}$ of dry plant. The absorbances for each methodology was determined using a microplate reader Synergy TM HT Multi-Mode.

\section{Antioxidant Assays 1,1-Diphenyl-2-Picrylhydrazyl Radical Free Radical Scavenging Activity}

The DPPH• $(3.9 \mathrm{ml}, 0.075 \mathrm{mM}$, in methanol) method reported by (Zeraik et al., 2016) was used to evaluate the antioxidant activity of $A$. copa. Briefly, $400 \mu \mathrm{l}$ of a solution, ( $2 \mathrm{mg} / \mathrm{ml}$ for standards), plus $2 \mathrm{ml}$ of DPPH solution were adjusted with the solvent methanol to an absorbance of $1.10 \pm$ 0.02 at $517 \mathrm{~nm}$. The mixture was homogenized using a vortex, allowed to react in the dark at room temperature for $20 \mathrm{~min}$, after which time absorbance was measured at $517 \mathrm{~nm}$. Gallic acid was used as a reference standard. The results were expressed as $\mathrm{IC}_{50}$ (concentration of infusion or standard in $\mu \mathrm{g} / \mathrm{ml}$ required to inhibit $50 \%$ of DPPH radical present in solution).

\section{Ferric Reduction Ability-Antioxidant Power Test}

For the Ferric Reducing Antioxidant Power (FRAP) test, the methodology determined by (Benzie and Strain, 1996) was performed with slight modifications (Morales and Paredes, 2014). The fresh working solution (FRAP reagent) included $300 \mathrm{mM}$ acetate buffer $\left(3.1 \mathrm{~g} \mathrm{C}_{2} \mathrm{H}_{3} \mathrm{NaO}_{2} \cdot 3 \mathrm{H}_{2} \mathrm{O}\right.$ and $16 \mathrm{ml}$ $\mathrm{CH}_{3} \mathrm{COOH} \quad \mathrm{pH}$ 3.6), $10 \mathrm{mM}$ TPTZ (2,4.6-tripyridyl-striazine) solution in $40 \mathrm{mM} \mathrm{HCl}$, and $20 \mathrm{mM} \mathrm{FeCl}_{3} \cdot 6 \mathrm{H}_{2} \mathrm{O}$ solution were prepared. Trolox was used as standard solution. Briefly, to some $100 \mu \mathrm{l}$ of the solution (or standards at $1 \mathrm{mg} / \mathrm{ml}$ ), $3 \mathrm{ml}$ of the FRAP solution was added and mixed using a vortex, allowed to react in the dark at room temperature for $5 \mathrm{~min}$. The absorbance measurement of the colored FeTPTZ complex was performed at $593 \mathrm{~nm}$. Absorbance values were replaced in the Trolox standard curve equation $(\mu \mathrm{mol} / \mathrm{l})$. The results were expressed as mg equivalents of Trolox/100 g of the dry plant.

\section{Oxygen Radical Absorbance Capacity}

The Oxygen radical absorbance capacity (ORAC) activity was determined according to the method previously reported by Huang with some modifications (Huang et al., 2002). Fluorescein $\left(3^{\prime}, 6^{\prime}\right.$-dihydroxyspiro[2-benzofuran-3,9'-xanthene]1-one) stock solution $(4 \mu \mathrm{M})$ was made in $75 \mathrm{mM}$ phosphate buffer $\mathrm{pH} 7.4$, stored at $4^{\circ} \mathrm{C}$ and used as the fluorescent probe (Zhang et al., 2009). AAPH (203.4 mg) was freshly prepared in $15 \mathrm{ml}$ of $75 \mathrm{mM}$ phosphate buffer and used to generate the peroxyl radical. Trolox was used as internal standard. In all the experiments, $150 \mu \mathrm{l}$ of fluorescein was added to each well. Blank wells received $25 \mu \mathrm{l}$ of phosphate buffer, standards $25 \mu \mathrm{l}$ of Trolox dilution, and samples $25 \mu \mathrm{l}$ of the sample. Excitation was monitored at $485 \mathrm{~nm}$ and emission at $528 \mathrm{~nm}$ with a $20 \mathrm{~nm}$ bandpass on an HTX Multi-Mode Microplate Reader. The results were obtained by quadratic regression equation (Trolox/samples vs. fluorescence decay curves) and expressed as $\mu \mathrm{M}$ Trolox equivalents per $100 \mathrm{~g}$ of the dry plant.

\section{Cholinesterase (ChE) Inhibition}

The infusions were evaluated by the Ellman's method, with some modifications (Ellman et al., 1961). Sample solution (50 $\mu \mathrm{l}, 2 \mathrm{mg} /$ $\mathrm{ml})$ was mixed with $120 \mu \mathrm{l}$ DTNB $(0.3 \mathrm{mM}, 5,5$-dithio-bis(2nitrobenzoic) acid) and $\mathrm{AChE}(0.026 \mathrm{U} / \mathrm{ml}, \mathrm{AChE}$ from Electric eel), or BChE $(0.026 \mathrm{U} / \mathrm{ml}, \mathrm{BChE}$ from horse serum) solution $(25 \mu \mathrm{l})$ in Tris- $\mathrm{HCl}$ buffer $50 \mathrm{mM}(\mathrm{pH} 8.0)$ in a 96-well microplate and incubated for $20 \mathrm{~min}$ at $37^{\circ} \mathrm{C}$. The reaction was then initiated with the addition of $20 \mu \mathrm{l}$ of ATCI $(1.5 \mathrm{mM}$, acetylthiocholine iodide) or BTCl (1.5 mM, butyrylthiocholine chloride). A blank was prepared for all reaction reagents without enzymes solution. The absorbances were recorded at $405 \mathrm{~nm}$ for $30 \mathrm{~min}$ at $37^{\circ} \mathrm{C}$ and the results were expressed as $\mathrm{IC}_{50}$ values (concentration range from 0.05 to $25 \mu \mathrm{g} / \mathrm{ml}$ ).

\section{Molecular Docking Studies}

The geometries and partial charges of major flavonoids apigenin7-O-glucoside, kaempferol-3-O-galactoside, kaempferol-3$O$-acetyl-glucoside and kaempferol-7-rhamnoside contained in A. copa infusions, as well as the known cholinesterase inhibitor galantamine, was fully optimized using the DFT method with the standard basis set PBE0/ 6-311 + $\mathrm{g}^{*}$ (Adamo and Barone, 1999). Molecular docking simulations over TcAChE and $h \mathrm{BChE}$ were performed using Autodock 4.2. All calculations were performed in Gaussian 09W software.

\section{Parasites and Cell Cultures}

T. cruzi (CL strain expressing beta-galactosidase) was used throughout this study (Andriani et al., 2011). Vero cells were infected with Tissue culture-derived trypomastigotes as previously described (San Francisco et al., 2017). Briefly, Vero cell monolayers were cultured in RPMI-1640 media supplemented with $10 \%$ fetal bovine sera. Culture flasks of $75 \mathrm{~cm}^{2}$ (Nunc, Eas YFlasks, Thermo Scientific, Bremen, Germany) containing subconfluent cultures of Vero cells, were infected with Tissue culture-derived trypomastigotes (CL strain) in a parasite/cell ratio equal to 5:1. After $3 \mathrm{~h}$ of infection, cell cultures were washed with PBS to remove parasites that did not penetrate into the cell and cultures were re-incubated in RPMI1640 medium at $37^{\circ} \mathrm{C}$ in a humidified $5 \% \mathrm{CO}_{2}$ atmosphere. Five days later, a pure population of T. cruzi trypomastigotes released in cell culture supernatants were harvested by centrifugation. $L$. amazonensis (IFLA/BR/67/PH8) was propagated as promastigotes at $26^{\circ} \mathrm{C}$ in M199 media supplemented with $5 \%$ 

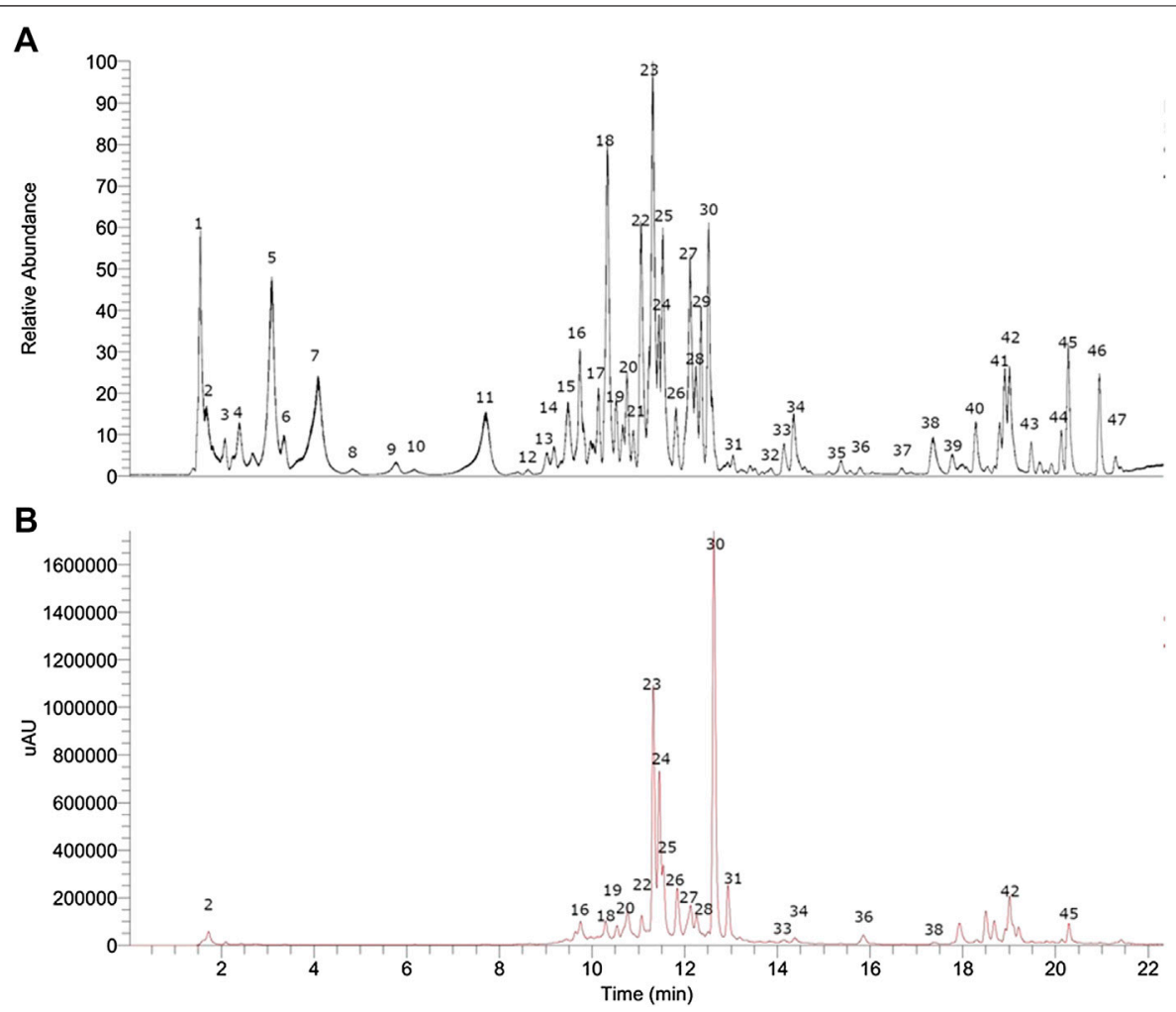

FIGURE 2 | UHPLC chromatograms of Artemisia copa Phil. (A) TIC chromatogram, (B) UV at $280 \mathrm{~nm}$ chomatogram.

penicillin/streptomycin, $0.1 \%$ hemin $(25 \mathrm{mg} / \mathrm{ml}$ in $0.1 \mathrm{~N} \mathrm{NaOH})$, $10 \mathrm{mM}$ adenine and $10 \%$ FBS, pH 7.5 as previously described (Sauter et al., 2019). Antiprotozoal activity against T. cruzi was evaluated as previously described (Gutiérrez et al., 2013). Briefly, serial dilutions of each infusion were performed in 96 wells microplates, in the range of 1000 to $0.48 \mu \mathrm{g} / \mathrm{ml}$ in a final volume of $100 \mu \mathrm{l}$. Then, $100 \mu \mathrm{l}$ of a suspension of trypomastigotes $1 \times 10^{7} \mathrm{ml}^{-1}$ were added and microplates were incubated $48 \mathrm{~h}$. Finally, a substrate, the $\beta$-Dgalactopyranoside was added, and the mixture incubated $4 \mathrm{~h}$ and finally, the enzymatic activity was recorded at $570 \mathrm{~nm}$ in a Tecan Infinite M200 Pro (Tecan, Männedorf, Switzerland). Leishmanicidal activity was evaluated as described (Loyola et al., 2001). Briefly, serial dilutions of each infusion were performed as described above. Then, $100 \mu \mathrm{l}$ of a suspension of $1 \times 10^{7}$ parasites $\mathrm{ml}^{-1}$ of $L$. amazonensis promastigotes were added and the microplates incubated $48 \mathrm{~h}$. Finally, the number of live parasites were count in a Neubauer chamber.

\section{Statistical Analysis}

The results are expressed as mean values \pm standard error using GraphPad Prism 8 (GraphPad software Corporation, La Jolla, CA, United States). One-way analysis of variance (ANOVA; repeating three times each measurement of sample solutions) $(p<0.05)$ was used for comparison of means.

\section{RESULTS AND DISCUSSION}

\section{Chemical Profiling}

In this study, 47 compounds were detected and tentatively identified in the infusion of $A$. copa (Figure 2). Previously, from a Argentinean sample of this plant $p$-coumaric acid, luteolin, chrysoeriol, and vitexin were reported, to present vasodilation (Gorzalczany et al., 2013b) also, some flavonoids were isolated from the sample: spinacetin, tricin, jaceosidin, axillarin, penduletin, and chrysoeriol which showed antiinflammatory activity (Moscatelli et al., 2006), the compound: 3,5-dihydroxy 6,7,3',4'-tetramethoxyflavone was isolated previously and presented antimicrobial activity (Morales et al., 2003). Several sesquiterpene lactones such as chrysartemin A, were also reported previously from this species (de la Fuente et al., 1986). In this work several compounds are reported for the first time in this species and the determination of those metabolites in this Chilean sample were characterized based on UV absorption and high-resolution mass spectrometry fragmentation patterns for the first time (Table 1). The content of individual compounds ( $\mathrm{mg} / \mathrm{Kg}$ of dry plant) are depicted in Supplementary Table S1 (Supplementary Material) and the chromatograms are depicted in Supplementary Figure S1 (Supplementary Material). Main compound peak $\mathbf{3 0}$ was not quantified, due to lack of standard. 
TABLE 1 | High-resolution UHPLC- MS Identification of metabolites from Artemisia copa infusion.

\begin{tabular}{|c|c|c|c|c|c|c|c|c|}
\hline $\begin{array}{l}\text { Peak } \\
\text { number }\end{array}$ & $\begin{array}{c}\text { UV max } \\
(\mathrm{nm})\end{array}$ & $\begin{array}{c}\text { Tentative } \\
\text { identification }\end{array}$ & $\begin{array}{l}\text { Formula } \\
{[\mathrm{M}-\mathrm{H}]^{-}}\end{array}$ & $\begin{array}{l}\text { Retention } \\
\text { time } \\
\text { (min) }\end{array}$ & $\begin{array}{c}\text { Theoretical } \\
\text { mass } \\
(\mathrm{m} / \mathrm{z})\end{array}$ & $\begin{array}{c}\text { Measured mass } \\
(\mathrm{m} / \mathrm{z})\end{array}$ & $\begin{array}{l}\text { Accuracy } \\
\text { (ppm) }\end{array}$ & $\begin{array}{c}\text { MS }^{n} \\
\text { ions } \\
\text { (ppm) }\end{array}$ \\
\hline 1 & 272-345 & Quinic acid & $\mathrm{C}_{7} \mathrm{H}_{11} \mathrm{O}_{6}^{-}$ & 1.55 & 191.05501 & 191.05569 & 3.56 & $173.04527\left(\mathrm{C}_{7} \mathrm{H}_{9} \mathrm{O}_{5}^{-}\right)\left(\mathrm{M}^{-}-\mathrm{H}_{2} \mathrm{O}\right) ; 109.02870\left(\mathrm{C}_{7} \mathrm{H}_{9} \mathrm{O}_{5}^{-}\right)$ \\
\hline 2 & 371 & Isoquinic acid & $\mathrm{C}_{7} \mathrm{H}_{11} \mathrm{O}_{6}^{-}$ & 1.69 & 191.05501 & 191.05557 & 2.92 & $111.00788\left(\mathrm{C}_{5} \mathrm{H}_{3} \mathrm{O}_{3}^{-}\right)$ \\
\hline 3 & 351 & Citric acid & $\mathrm{C}_{6} \mathrm{H}_{7} \mathrm{O}_{7}^{-}$ & 2.07 & 191.01863 & 191.01929 & 3.44 & $111.00790\left(\mathrm{C}_{5} \mathrm{H}_{3} \mathrm{O}_{3}^{-}\right)$ \\
\hline 4 & - & 2-Hydroxyglutaric acid & $\mathrm{C}_{5} \mathrm{H}_{7} \mathrm{O}_{5}$ & 2.39 & 147.02880 & 147.02917 & 2.55 & $133.01477\left(\mathrm{C}_{4} \mathrm{H}_{5} \mathrm{O}_{5}{ }^{-}\right)$ \\
\hline 5 & - & Lilioside A & $\mathrm{C}_{11} \mathrm{H}_{19} \mathrm{O}_{9}^{-}$ & 3.08 & 295.10236 & 295.10349 & 3.81 & $133.04996\left(\mathrm{C}_{5} \mathrm{H}_{9} \mathrm{O}_{4}^{-}\right)$ \\
\hline 6 & 246 & 2,3-Butanediol apiosylglucoside & $\mathrm{C}_{15} \mathrm{H}_{27} \mathrm{O}_{11}^{-}$ & 3.34 & 383.15479 & 383.15598 & 3.09 & $129.09160\left(\mathrm{C}_{7} \mathrm{H}_{13} \mathrm{O}_{2}^{-}\right)$ \\
\hline 7 & 231 & Artemisinic acid & $\mathrm{C}_{15} \mathrm{H}_{21} \mathrm{O}_{2}^{-}$ & 4.09 & 233.15361 & 233.15446 & 3.68 & $161.04453\left(\mathrm{C}_{6} \mathrm{H}_{9} \mathrm{O}_{5}^{-}\right)$ \\
\hline 8 & 299 & 2,4,6-Trihydroxyanisole & $\mathrm{C}_{7} \mathrm{H}_{7} \mathrm{O}_{4}^{-}$ & 4.85 & 155.03389 & 155.03441 & 3.37 & $129.09145\left(\mathrm{C}_{7} \mathrm{H}_{13} \mathrm{O}_{2}^{-}\right)$ \\
\hline 9 & 228 & Cinnamyl tiglate & $\mathrm{C}_{14} \mathrm{H}_{15} \mathrm{O}_{2}^{-}$ & 5.77 & 215.10666 & 215.10745 & 3.70 & $197.09648\left(\mathrm{C}_{14} \mathrm{H}_{13} \mathrm{O}^{-}\right)$ \\
\hline 10 & - & Pleoside & $\mathrm{C}_{15} \mathrm{H}_{19} \mathrm{O}_{9}^{-}$ & 6.12 & 343.10346 & 343.10361 & 3.63 & - \\
\hline 11 & - & Methyl vanillate glucoside & $\mathrm{C}_{15} \mathrm{H}_{19} \mathrm{O}_{9}^{-}$ & 7.71 & 343.10236 & 343.10352 & 3.37 & - \\
\hline 12 & $217-281$ & Rehmaionoside A & $\mathrm{C}_{19} \mathrm{H}_{33} \mathrm{O}_{8}^{-}$ & 8.64 & 389.21809 & 389.21957 & 3.38 & $181.05011\left(\mathrm{C}_{9} \mathrm{H}_{9} \mathrm{O}_{4}^{-}\right)$ \\
\hline 13 & $221-284$ & Gaultherin & $\mathrm{C}_{19} \mathrm{H}_{25} \mathrm{O}_{12}^{-}$ & 9.01 & 445.13405 & 445.13528 & 2.76 & $374.16104,199.09721$ \\
\hline 14 & $222-285$ & 4-Hydroxybenzoic acid & $\mathrm{C}_{7} \mathrm{H}_{5} \mathrm{O}_{3}^{-}$ & 9.19 & 137.02442 & 137.02379 & 3.41 & - \\
\hline 15 & - & 2-Isopropylmalic acid & $\mathrm{C}_{7} \mathrm{H}_{11} \mathrm{O}_{5}^{-}$ & 9.48 & 175.06010 & 175.06079 & 3.94 & - \\
\hline 16 & 218-325 & Chlorogenic acid & $\mathrm{C}_{16} \mathrm{H}_{17} \mathrm{O}_{9}^{-}$ & 9.74 & 353.08781 & 353.08789 & 3.34 & $191.05571\left(\mathrm{C}_{7} \mathrm{H}_{11} \mathrm{O}_{6}^{-}\right)$ \\
\hline 17 & 223-409 & 7-Demethylsuberosin & $\mathrm{C}_{14} \mathrm{H}_{13} \mathrm{O}_{3}^{-}$ & 10.13 & 229.08592 & 229.08670 & 3.40 & $135.04437\left(\mathrm{C}_{8} \mathrm{H}_{7} \mathrm{O}_{2}^{-}\right)$ \\
\hline 18 & $270-329-427$ & Dihydro-p-coumaroylglucose & $\mathrm{C}_{15} \mathrm{H}_{19} \mathrm{O}_{8}^{-}$ & 10.33 & 327.10744 & 327.10855 & 3.47 & $147.04445\left(\mathrm{C}_{9} \mathrm{H}_{7} \mathrm{O}_{2}^{-}\right)$ \\
\hline 19 & 259-431 & Schaftoside & $\mathrm{C}_{26} \mathrm{H}_{27} \mathrm{O}_{14}^{-}$ & 10.52 & 563.13953 & 563.14020 & 1.18 & 374.16104 \\
\hline 20 & 269-346 & Kaempferol-3-O-glucoside & $\mathrm{C}_{21} \mathrm{H}_{19} \mathrm{O}_{11}$ & 10.77 & 447.09219 & 447.09329 & 2.47 & 175.03952 \\
\hline 21 & $226-330$ & Feruloyl arabinobiose & $\mathrm{C}_{20} \mathrm{H}_{25} \mathrm{O}_{12}^{-}$ & 10.88 & 457.13405 & 457.13535 & 2.82 & $215.10750\left(\mathrm{C}_{14} \mathrm{H}_{15} \mathrm{O}_{2}^{-}\right)$ \\
\hline 22 & $218-326$ & 3-O-Feruloylquinic acid & $\mathrm{C}_{17} \mathrm{H}_{19} \mathrm{O}_{9}^{-}$ & 11.06 & 367.10236 & 367.10364 & 3.48 & $173.04486\left(\mathrm{C}_{7} \mathrm{H}_{9} \mathrm{O}_{5}^{-}\right)$ \\
\hline 23 & 213-338 & Apigenin 7-O-glucoside & $\mathrm{C}_{21} \mathrm{H}_{19} \mathrm{O}_{10^{-}}$ & 11.30 & 431.09727 & 431.09845 & 2.74 & $197.09671\left(\mathrm{C}_{14} \mathrm{H}_{13} \mathrm{O}^{-}\right)$ \\
\hline 24 & $225-309-$ & Kaempferol 7-rhamnoside & $\mathrm{C}_{21} \mathrm{H}_{19} \mathrm{O}_{10}-$ & 11.44 & 431.09727 & 431.09851 & 2.87 & $163.03955\left(\mathrm{C}_{9} \mathrm{H}_{7} \mathrm{O}_{3}^{-}\right)$ \\
\hline 25 & 268-342 & Kaempferol-3-O-galactoside & $\mathrm{C}_{21} \mathrm{H}_{19} \mathrm{O}_{11}^{-}$ & 11.52 & 447.09219 & 447.09341 & 2.74 & $285.04037\left(\mathrm{C}_{15} \mathrm{H}_{9} \mathrm{O}_{6}^{-} ;\right.$kaempferol) \\
\hline 26 & 296-439 & Cynarine & $\mathrm{C}_{25} \mathrm{H}_{23} \mathrm{O}_{12}{ }^{-}$ & 11.81 & 515.11840 & 515.11932 & 1.78 & $135.04436\left(\mathrm{C}_{8} \mathrm{H}_{7} \mathrm{O}_{2}^{-}\right)$ \\
\hline 27 & $265-354$ & Kaempferol-3-O-acetyl-glucoside & $\mathrm{C}_{23} \mathrm{H}_{21} \mathrm{O}_{12}^{-}$ & 12.12 & 489.10275 & 489.10376 & 2.05 & $285.04028\left(\mathrm{C}_{15} \mathrm{H}_{9} \mathrm{O}_{6}^{-} ;\right.$Kaempferol $)$ \\
\hline 28 & $336-462$ & Nepochlorogenic acid 1,3 di-O-caffeoyl quinic acid & $\mathrm{C}_{25} \mathrm{H}_{23} \mathrm{O}_{12}^{-}$ & 12.23 & 515.11840 & 515.11945 & 2.03 & $191.05562\left(\mathrm{C}_{7} \mathrm{H}_{11} \mathrm{O}_{6}^{-}\right)$ \\
\hline 29 & $231-332-462$ & Hydroxyoctanoic acid-O-Glucoside & $\mathrm{C}_{14} \mathrm{H}_{25} \mathrm{O}_{8}^{-}$ & 12.33 & 321.15439 & 321.15564 & 3.87 & $159.10207\left(\mathrm{C}_{8} \mathrm{H}_{15} \mathrm{O}_{3}^{-} ;\right.$hydroxyoctanoic acid $)$ \\
\hline 30 & $250-326$ & Chrysartemin A & $\mathrm{C}_{15} \mathrm{H}_{17} \mathrm{O}_{5}^{-}$ & 12.51 & 277.10705 & 277.10809 & 3.75 & $135.08081\left(\mathrm{C}_{9} \mathrm{H}_{11} \mathrm{O}^{-}\right)$ \\
\hline 31 & 236 & Unknown & $\mathrm{C}_{13} \mathrm{H}_{13} \mathrm{O}_{15}$ & 13.05 & 409.02599 & 409.02380 & -2.68 & $181.08667\left(\mathrm{C}_{10} \mathrm{H}_{13} \mathrm{O}_{3}^{-}\right)$ \\
\hline 32 & $228-427$ & trans- $\beta$-Damascenone & $\mathrm{C}_{13} \mathrm{H}_{17} \mathrm{O}^{-}$ & 13.87 & 189.12739 & 189.12820 & 4.29 & $145.02859\left(\mathrm{C}_{9} \mathrm{H}_{5} \mathrm{O}_{2}^{-}\right)$ \\
\hline 33 & $232-309$ & Unknown & $\mathrm{C}_{13} \mathrm{H}_{15} \mathrm{O}_{4}^{-}$ & 14.14 & 235.09649 & 235.09746 & 4.13 & $145.02879\left(\mathrm{C}_{9} \mathrm{H}_{5} \mathrm{O}_{2}^{-}\right)$ \\
\hline 34 & $265-354$ & Kaempferol & $\mathrm{C}_{15} \mathrm{H}_{19} \mathrm{O}_{6}^{-}$ & 14.36 & 285.03936 & 285.04059 & 4.29 & $135.04439\left(\mathrm{C}_{8} \mathrm{H}_{7} \mathrm{O}_{2}^{-}\right)$ \\
\hline 35 & $239-340$ & 3,7-Dimethylquercetagetin & $\mathrm{C}_{17} \mathrm{H}_{13} \mathrm{O}_{8}^{-}$ & 15.37 & 345.06049 & 345.06183 & 3.89 & $129.09175\left(\mathrm{C}_{7} \mathrm{H}_{13} \mathrm{O}_{2}^{-}\right)$ \\
\hline 36 & 290-396 & 4-Propionylbenzoic acid & $\mathrm{C}_{10} \mathrm{H}_{9} \mathrm{O}_{3}^{-}$ & 15.78 & 177.05462 & 177.05531 & 3.91 & $145.02884\left(\mathrm{C}_{9} \mathrm{H}_{5} \mathrm{O}_{2}^{-}\right)$ \\
\hline 37 & 239-382 & Peuarenarine & $\mathrm{C}_{24} \mathrm{H}_{25} \mathrm{O}_{8}^{-}$ & 16.68 & 441.15439 & 441.15570 & 2.96 & $145.02876\left(\mathrm{C}_{9} \mathrm{H}_{5} \mathrm{O}_{2}^{-}\right)$ \\
\hline 38 & $237-345$ & 7, 3'-dimethoxyquercetin & $\mathrm{C}_{17} \mathrm{H}_{13} \mathrm{O}_{7}^{-}$ & 17.36 & 329.06558 & 329.06683 & 3.81 & $299.01971\left(\mathrm{C}_{15} \mathrm{H}_{7} \mathrm{O}_{7}^{-}\right.$; quercetin) \\
\hline 39 & $233-340$ & 3-O-Methylkaempferol & $\mathrm{C}_{16} \mathrm{H}_{11} \mathrm{O}_{6}^{-}$ & 17.77 & 299.05501 & 299.05627 & 4.21 & $134.03671\left(\mathrm{C}_{8} \mathrm{H}_{6} \mathrm{O}_{2}^{-}\right)$ \\
\hline 40 & $255-367$ & Eupatolitin & $\mathrm{C}_{17} \mathrm{H}_{13} \mathrm{O}_{8}^{-}$ & 18.28 & 345.06159 & 345.06189 & 4.04 & $183.08127\left(\mathrm{C}_{13} \mathrm{H}_{11} \mathrm{O}^{-}\right)$ \\
\hline 41 & 256-349 & Irigenin & $\mathrm{C}_{18} \mathrm{H}_{15} \mathrm{O}_{8}^{-}$ & 18.90 & 359.07724 & 359.07748 & 3.70 & $329.02969\left(\mathrm{C}_{16} \mathrm{H}_{9} \mathrm{O}_{8}^{-}\right)$ \\
\hline 42 & $262-425$ & Myristicin & $\mathrm{C}_{11} \mathrm{H}_{11} \mathrm{O}_{3}^{-}$ & 19.01 & 191.07027 & 191.07101 & 3.89 & $145.02888\left(\mathrm{C}_{9} \mathrm{H}_{5} \mathrm{O}_{2}^{-}\right)$ \\
\hline 43 & $241-312$ & Calodendrolide & $\mathrm{C}_{15} \mathrm{H}_{15} \mathrm{O}_{4}^{-}$ & 19.47 & 259.09758 & 259.09753 & 4.04 & $145.02895\left(\mathrm{C}_{9} \mathrm{H}_{5} \mathrm{O}_{2}^{-}\right)$ \\
\hline 44 & 269-338 & Eupatorin & $\mathrm{C}_{18} \mathrm{H}_{15} \mathrm{O}_{7}^{-}$ & 20.14 & 343.08123 & 343.08264 & 4.11 & $215.10728\left(\mathrm{C}_{14} \mathrm{H}_{15} \mathrm{O}_{2}^{-}\right)$ \\
\hline 45 & $254-354$ & 3,6,7,8,3'-tetramethoxymyricetin & $\mathrm{C}_{19} \mathrm{H}_{17} \mathrm{O}_{8}^{-}$ & 20.27 & 373.09289 & 373.09320 & 3.76 & $129.09129\left(\mathrm{C}_{7} \mathrm{H}_{13} \mathrm{O}_{2}^{-}\right)$ \\
\hline 46 & 248-392 & Pentyl benzoate & $\mathrm{C}_{12} \mathrm{H}_{15} \mathrm{O}_{2}^{-}$ & 20.95 & 191.10666 & 191.10741 & 3.94 & $427.21265\left(\mathrm{C}_{25} \mathrm{H}_{31} \mathrm{O}_{6}^{-}\right)$ \\
\hline 47 & $291-396$ & 5,7-Dimethoxy-2,2-dimethylchromene & $\mathrm{C}_{13} \mathrm{H}_{15} \mathrm{O}_{3}^{-}$ & 21.30 & 219.10157 & 219.10248 & 4.14 & $145.02876\left(\mathrm{C}_{9} \mathrm{H}_{5} \mathrm{O}_{2}^{-}\right)$ \\
\hline
\end{tabular}




\section{Sugars and Hydrocarbons}

Peak 1 with a pseudo-molecular ion at $m / z: 191.05569$ was identified as quinic acid $\left(\mathrm{C}_{7} \mathrm{H}_{11} \mathrm{O}_{6}{ }^{-}\right)$and its isomer peak 2 as isoquinic acid at $\mathrm{m} / z$ 191.05557. Peak 3 was identified as citric acid $\left(\mathrm{C}_{6} \mathrm{H}_{7} \mathrm{O}_{7}^{-}\right)$. Peak 4 with a molecular ion at $\mathrm{m} / z$ : 47.02917 was identified as 2-hydroxyglutaric acid $\left(\mathrm{C}_{5} \mathrm{H}_{7} \mathrm{O}_{5}{ }^{-}\right)$. peak 12 as rehmaionoside $\mathrm{A}\left(\mathrm{C}_{19} \mathrm{H}_{33} \mathrm{O}_{8}{ }^{-}\right)$, a constituent of Rehmannia glutinosa (Gaertn.) DC. (Liu et al., 2020).

\section{Phenolic Acids}

Peak 5 with a $[\mathrm{M}-\mathrm{H}]^{-}$ion at $m / z: 295.10349$ was identified as the coumaroyl glycerol lilioside A $\left(\mathrm{C}_{11} \mathrm{H}_{19} \mathrm{O}_{9}{ }^{-}\right)$(Kaneda, 1990), peak 6 was identified as 2,3-butanediol apiosylglucoside (383.15598, $\mathrm{C}_{15} \mathrm{H}_{27} \mathrm{O}_{11}{ }^{-}$). Peak 8 with a $[\mathrm{M}-\mathrm{H}]^{-}$ion at $\mathrm{m} / z 155.03441$ was identified as 2,4,6-trihydroxyanisole $\left(\mathrm{C}_{7} \mathrm{H}_{7} \mathrm{O}_{4}^{-}\right)$. Peak 9 was identified as cinnamyl tiglate $\left(\mathrm{C}_{14} \mathrm{H}_{15} \mathrm{O}_{2}{ }^{-}\right)$, peak 10 with a molecular ion at $m / z$ : 343.10361, was identified as pleoside $\left(\mathrm{C}_{15} \mathrm{H}_{19} \mathrm{O}_{9}{ }^{-}\right)$this compound was reported antiproliferative together with chlorogenic acid (Jafari et al., 2017), peak 11 as its isomer methyl vanillate glucoside, Peak $\mathbf{1 3}$ as gaultherin $\left(\mathrm{C}_{19} \mathrm{H}_{25} \mathrm{O}_{12}{ }^{-}\right)$. Peak 14 with a $[\mathrm{M}-\mathrm{H}]^{-}$ion at $\mathrm{m} / \mathrm{z}$ : 137.02379 was identified as 4-hydroxybenzoic acid $\left(\mathrm{C}_{7} \mathrm{H}_{5} \mathrm{O}_{3}{ }^{-}\right)$, peak 15 as 2-isopropylmalic acid $\left(\mathrm{C}_{7} \mathrm{H}_{11} \mathrm{O}_{5}{ }^{-}\right)$. Peak 19 as schaftoside $\left(\mathrm{C}_{26} \mathrm{H}_{27} \mathrm{O}_{14}{ }^{-}\right)$peak 16 and 28 were identified as isomers of chlorogenic acid $\left(\mathrm{C}_{16} \mathrm{H}_{17} \mathrm{O}_{9}{ }^{-}\right)$, peak 17 as 7-demethylsuberosin $\left(\mathrm{C}_{14} \mathrm{H}_{13} \mathrm{O}_{3}^{-}\right)$, and peak 18 as dihydro- $p$-coumaroylglucose $\left(\mathrm{C}_{15} \mathrm{H}_{19} \mathrm{O}_{8}{ }^{-}\right)$, respectively. Peak 21 as feruloyl arabinobiose (457.13535), Peak 22 with a $[\mathrm{M}-\mathrm{H}]^{-}$ion at $\mathrm{m} / z$ : 367.10364 was identified as 3-O-feruloylquinic acid $\left(\mathrm{C}_{17} \mathrm{H}_{19} \mathrm{O}_{9}{ }^{-}\right)$, peak 26 as cynarine, peak 32 as trans- $\beta$-damascenone $\left(\mathrm{C}_{13} \mathrm{H}_{17} \mathrm{O}^{-}\right)$, and peak 36 as 4-propionylbenzoic acid (177.05531) and 37 as peuarenarine $\left(\mathrm{C}_{24} \mathrm{H}_{25} \mathrm{O}_{8}{ }^{-}\right)$, peak 46 as pentyl benzoate $\left(\mathrm{C}_{12} \mathrm{H}_{15} \mathrm{O}_{2}{ }^{-}\right)$and finally, peak 47 as 5,7-dimethoxy-2,2dimethylchromene $\left(\mathrm{C}_{13} \mathrm{H}_{15} \mathrm{O}_{3}{ }^{-}\right)$.

\section{Fatty Acids}

Peak 29 was identified as hydroxyoctanoic acid-O-glucoside $\left(\mathrm{C}_{14} \mathrm{H}_{25} \mathrm{O}_{8}{ }^{-}\right)$

\section{Flavonoids}

Peak 20 with a $[\mathrm{M}-\mathrm{H}]^{-}$ion at $m / z: 447.09372$ was identified as kaempferol-3-O-glucoside $\left(\mathrm{C}_{21} \mathrm{H}_{19} \mathrm{O}_{11}{ }^{-}\right)$while peak 23 with a pseudo-molecular ion at $\mathrm{m} / z \quad 431.09845$ was identified as apigenin 7-O-glucoside $\left(\mathrm{C}_{21} \mathrm{H}_{19} \mathrm{O}_{10}{ }^{-}\right)$(Taşkın et al., 2018) and peak 24 as kaempferol 7-rhamnoside $\left(\mathrm{C}_{21} \mathrm{H}_{19} \mathrm{O}_{10}{ }^{-}\right)$. Peak 25 as kaempferol-3-O-galactoside $\left(\mathrm{C}_{21} \mathrm{H}_{19} \mathrm{O}_{11}{ }^{-}\right)$and peak 27 with a molecular ion at $m / z: 489.10376$ as kaempferol-3-O-acetylglucoside $\left(\mathrm{C}_{23} \mathrm{H}_{21} \mathrm{O}_{12}{ }^{-}\right)$a constituent of Moringa oleifera Lam. leaves (Mousa et al., 2019), finally, peaks 34, 35 and 38-42 and 44, 45 as: kaempferol $\left(\mathrm{C}_{15} \mathrm{H}_{19} \mathrm{O}_{6}{ }^{-}\right)$, 3,7-dimethylquercetagetin $\left(\mathrm{C}_{17} \mathrm{H}_{13} \mathrm{O}_{8}^{-}\right)$, 7,3'-dimethoxyquercetin $\quad\left(\mathrm{C}_{17} \mathrm{H}_{13} \mathrm{O}_{7}^{-}\right)$, 3-Omethylkaempferol $\left(\mathrm{C}_{16} \mathrm{H}_{11} \mathrm{O}_{6}^{-}\right)$, eupatolitin $\left(\mathrm{C}_{17} \mathrm{H}_{13} \mathrm{O}_{8}^{-}\right)$, irigenin $\left(\mathrm{C}_{18} \mathrm{H}_{15} \mathrm{O}_{8}{ }^{-}\right)$, myristicin $\left(\mathrm{C}_{11} \mathrm{H}_{11} \mathrm{O}_{3}{ }^{-}\right)$, eupatorin $\left(\mathrm{C}_{18} \mathrm{H}_{15} \mathrm{O}_{7}{ }^{-}\right)$, and 3,6,7,8,3'-tetramethoxymyricetin $\left(\mathrm{C}_{19} \mathrm{H}_{17} \mathrm{O}_{8}{ }^{-}\right)$, respectively.
TABLE 2 | Total phenolics, total flavonoids, and antioxidants activity of Artemisia copa infusion

\begin{tabular}{lcc} 
Assay & Artemisia copa infusion & Standard \\
\hline Total phenolics $^{\mathrm{a}}$ & $155.6 \pm 2.9$ & - \\
Total flavonoids $^{\mathrm{b}}$ & $5.5 \pm 0.2$ & - \\
FRAP $^{\mathrm{c}}$ & $6.2 \pm 0.2$ & - \\
ORAC $^{\mathrm{d}}$ & $1989 \pm 5.2$ & - \\
DPPH $^{\mathrm{e}}$ & $89.72 \pm 0.01$ & Gallic acid: $0.55 \pm 0.01$
\end{tabular}

All values were expressed as means \pm SEM $(\mathrm{n}=3)$.

FRAP, ferric reducing/antioxidant power; ORAC, Oxygen Radical Absorbance Capacity; $D P P H, 2,2-d i p h e n y l-1$-picryl-hydrazyl-hydrate. All values in the column are significantly different (at $\mathrm{p}<0.05$ )

aexpressed in $\mathrm{mg}$ gallic acid equivalent per $\mathrm{g}$ of dry plant.

${ }^{b}$ expressed in $\mathrm{mg}$ quercetin per $g$ of dry plant.

${ }^{c}$ expressed in $\mathrm{mg}$ trolox equivalent per $100 \mathrm{~g}$ of the dry plant.

${ }^{d}$ expressed in $\mu M$ Trolox equivalents per $100 \mathrm{~g}$ of the dry plant.

expressed as $I C_{50}$ in $\mu \mathrm{g}$ of extract or standard per $\mathrm{ml}$.

\section{Sesquiterpene Lactones}

Artemisinic acid peak $7\left(\mathrm{C}_{15} \mathrm{H}_{21} \mathrm{O}_{2}^{-}\right)$, main compound in famous Chinese medicinal plant Artemisia annua L. (Bora and Sharma, 2011), was found and identified with a molecular ion at $\mathrm{m} / z$ : 233.15446, and peak 30 as chrysartemin $\mathrm{A}\left(\mathrm{C}_{15} \mathrm{H}_{17} \mathrm{O}_{5}{ }^{-}\right)$also constituent of the Artemisia genus.

\section{Total Phenolic Content, Total Flavonoid Content, and Antioxidant Activity}

Artemisia copa infusions were assessed in vitro for total phenolic content (TPC) and total flavonoid content (TFC) also, DPPH, FRAP, and ORAC assays were used to determine the antioxidant properties and are summarized in Table 2 . These in vitro assays are simple and widely employed for the evaluation of antioxidant power and ROS quenching (Moon and Shibamoto, 2009). These results were compared to another very used Andean altiplano plant, Aloysia deserticola (Phil.) Lu-Irving and O'Leary (syn. Acantholippia deserticola Phil.) (Verbenaceae) previously reported by us (Larrazabal et al., 2018). Phenolic compounds are structurally diverse and comprise phenolic acids, flavonoids, tannins, coumarins, chalcones, iridoids, among others, most of them bearing phenolic moieties that give them antioxidant and beneficial health properties. In this study three main classes of compounds were tentatively identified: organic acids, feruloyl and coumaroyl derivatives, plus flavonoids. In addition, 47 compounds were detected in their corresponding infusion, that could be responsible for the bioactivities reported. The results observed for TPC and TFC of $A$. copa infusion were $155.6 \pm$ $2.9 \mathrm{mg} / \mathrm{g}$ and the $5.5 \pm 0.2 \mathrm{mg} / \mathrm{g}$ of dry plant, respectively. On the other hand, DPPH was performed to know the bleaching capacities of the infusion, and showed an $\mathrm{IC}_{50}$ of $89.72 \pm 0.01 \mu \mathrm{g} /$ $\mathrm{ml}$ infusion. While the ABTS and DPPH values for A. deserticola infusions were 337 and $438 \mu \mathrm{g} / \mathrm{ml}$ (Larrazabal et al., 2018). Also, the FRAP assay was conducted giving the lower antioxidant power obtained $(6.2 \pm 0.2 \mathrm{mg}$ trolox equivalent per $100 \mathrm{~g}$ of the dry plant.) compared to the famous infusions of the arid plant A. deserticola (Larrazabal et al., 2018). This assay is based on the ability to reduce $\mathrm{Fe}^{+3}$ to $\mathrm{Fe}^{+2}$ by antioxidants in a sample. Regarding the ORAC assay, the infusion value was $1989 \pm$ 
TABLE 3 | Cholinesterase inhibitory activity of Artemisia copa infusion.

\begin{tabular}{lcc}
\hline Assay & Artemisia copa infusion $\left(\mathbf{I C}_{\mathbf{5 0}}\right)$ & Galantamine \\
\hline (IC & \\
AChE & $3.92 \pm 0.08$ & $0.26 \pm 0.03$ \\
BChE & $44.13 \pm 0.10$ & $3.82 \pm 0.02$
\end{tabular}

All values were expressed as means \pm SEM $(\mathrm{n}=3) .1 C_{50}$ as expressed in $\mu \mathrm{g}$ per $\mathrm{ml}$. AChE, Acetylcholinesterase; BChE, Butyrylcholinesterase. Values in the same column marked with the same letter are not significantly different (at $\mathrm{p}<0.05$ ).

$5.2 \mu \mathrm{M}$ Trolox equivalents (TEAC) per $100 \mathrm{~g}$ of the dry plant. These values can classify these $A$. copa infusions as moderate antioxidants properties, like edible fruits such as cherries, strawberries, and plums (Rodríguez et al., 2014; Ramirez et al., 2015).

\section{Cholinesterase Inhibitory Activity}

In this study, Artemisia copa infusion was assessed for in vitro inhibitory activity against $\mathrm{AChE}$ and $\mathrm{BChE}$ using galantamine as the positive control (reference: galantamine $0.26 \pm 0.03 \mu \mathrm{g} / \mathrm{ml}$ ). The results are summarized in Table 3 and are expressed as $\mathrm{IC}_{50}$ values $(\mu \mathrm{g} / \mathrm{ml})$. The inhibition of key enzymes has been a very useful strategy to know the pharmacological potential of herbal medicines. Moreover, inhibition of enzymes linked to Alzheimer's disease (AD), such AChE and BChE (Pan et al., 2013). In the AChE assays, $A$. copa showed an $\mathrm{IC}_{50}$ of $3.92 \pm$ $0.08 \mu \mathrm{g} / \mathrm{ml}$, while in the $\mathrm{BChE}$ assays the $\mathrm{IC}_{50}$ results were $44.13 \pm 0.10 \mu \mathrm{g} / \mathrm{ml}$ respectively. In previous reports the cholinesterase inhibitory activity of Algerian Artemisia spp: A. absinthium L., A. fragrans Willd. and A. herba-alba Asso were evaluated and showed similar results against AChE but not activity against BChE (Orhan et al., 2010), also Turkish Artemisia, A. absinthium was reported (Zengin et al., 2017). A. copa possessed the capacity to inhibit AChE and BChE enzymes.

Regarding the constituents identified in the infusion of $A$. $\operatorname{copa}$, the literature has indicated that, for example, the increase of brain AChE activity was reported for citric acid, acting as a neuroprotective agent in animal models (Abdel-Salam et al., 2016). Regarding hydroxybenzoic acid, have evaluated the antioxidants and ChE inhibitors properties of hydroxybenzoic acid derivatives as dual-targets (Oliveira et al., 2018). In a previous report phenolic acids from malt, included hydroxybenzoic acid, showed efficient properties against AChE and BChE respectively (Szwajgier and Borowiec, 2012). Chlorogenic acid exert protective effects on learning and memory impairment. Furthermore, ex vivo experiments showed inhibition of AChE activity in hippocampus and frontal cortex. On the other hand, in vitro experiments inhibit AChE activity with a $\mathrm{IC}_{50}=98.17 \mu \mathrm{g} / \mathrm{ml}$ (Kwon et al., 2010). Also, the combination of chlorogenic acid with caffeic acid displayed an antagonist response against AChE and BChE. The neuroprotective properties have been associated to the $\mathrm{ChE}$ inhibition, as well to the prevention of oxidative damage (Oboh et al., 2013). AChE activity have been reported for 7demethylsuberosin isolated from genus Angelica, suggesting a potential agent for the AD (Kang et al., 2001; Kwon et al., 2017). In addition, Schaftoside isolated from the water layer of Lycopodiella cernua (L.) Pic.Serm. showed strong inhibitory activity against BChE (Hung et al., 2015).
The in vitro ChE inhibitory activities of different flavonoids derivatives (rutin, quercetin and kaempferol-3-O-galactoside) have been previously investigated. Docking experiments of quercetin showed a competitive inhibition mechanism against both enzymes (Khan et al., 2009). The principal active compound from Cynara scolymus L., cynarine, was purified from Onopordum illyricum L. and showed antioxidant, antiradical and inhibitory activities against AChE (Topal et al., 2016). On the other hand, the isoflavonoid irigenin was tested against $\mathrm{AChE}$ and $\mathrm{BChE}$. The results showed a moderate activity against $\mathrm{BChE}$ (less to $40 \%$ of inhibition) (Ślusarczyk et al., 2019). Besides, inhibitory activity of AChE of myristicin have been investigated in vitro and in vivo experiments. The enzymatic results indicate that myristicin caused competitive and noncompetitive inhibition (Jaiswal et al., 2010). Finally, the liminoid calodrendolide showed neuroprotective effects against glutamate-induced neurotoxicity in rat cortical cells (Jeong et al., 2008).

This result suggests that infusion from $A$. copa could be explored as an alternative medicine for $\mathrm{AD}$.

\section{Molecular Docking Studies}

To get insights on the intermolecular interactions, four flavonoids contained in the Artemisia copa infusion: apigenin-7$O$-glucoside, kaempferol-3-O-galactoside, kaempferol-3$O$-acetyl-glucoside and kaempferol-7-rhamnoside (which are in high amount and proportion in this species), as well as the known cholinesterase inhibitor galantamine (Supplementary Figure S2), were selected and subjected to molecular docking studies over AChE (TcAChE) and BChE ( $h \mathrm{BChE}$ ) (Full description, Supplementary Material). The relationship between flavonoids and their potential as cholinesterase inhibitors have been reported (Sevindik et al., 2015; Khan et al., 2018). Table 4 shows the binding energies expressed in $\mathrm{kcal} / \mathrm{mol}$ of every compound mentioned above.

In TcAChE the binding energy descriptors for flavonoids kaempferol-3-O-galactoside and kaempferol-3-O-acetylglucoside turned out to be better than for flavonoids apigenin7-O-glucoside and kaempferol-7-rhamnoside. Apigenin-7$O$-glucoside, as well as kaempferol-7-rhamnoside displayed deficient binding energies of $9.76 \mathrm{kcal} / \mathrm{mol}$ and $12.66 \mathrm{kcal} / \mathrm{mol}$ respectively, compared to $-2.04 \mathrm{kcal} / \mathrm{mol}$ of kaempferol-3$O$-galactoside and $-1.27 \mathrm{kcal} / \mathrm{mol}$ of kaempferol-3-O-acetylglucoside, suggesting that the inhibitory activity over the enzyme lies predominantly in the first two flavonoids mentioned above. This could be explained due flavonoids kaempferol-3-O-galactoside and kaempferol-3-O-acetylglucoside are arranged in similar manners into the catalytic site of the enzyme. In the same way, apigenin-7-O-glucoside and kaempferol-7-rhamnoside are also closely overlapped between them. Therefore, both pairs of compounds carry out different interactions with the amino acids, although derivatives kaempferol-3-O-galactoside and apigenin-7-O-glucoside performed hydrogen bond interactions with the amino acid Glu199 (Figure 3). The differences in the abilities to inhibit the enzyme and the differences in the binding energies of the four flavonoids, must be due because of the greatest amount of 
TABLE 4 | Binding energies obtained from docking experiments of selected flavonoids and Galantamine over cholinesterases $T C A C h E$ and $h$ BChE.

\section{Compounds}

Binding energy (kcal/mol) Acetylcholinesterase (TcAChE)
Binding energy (kcal/mol) Butyrylcholinesterase ( $h B C h E)$

9.76
-2.04
-1.27
12.66
-11.81

$-5.93$

$-8.92$

$-8.36$

$-3.38$

$-9.50$
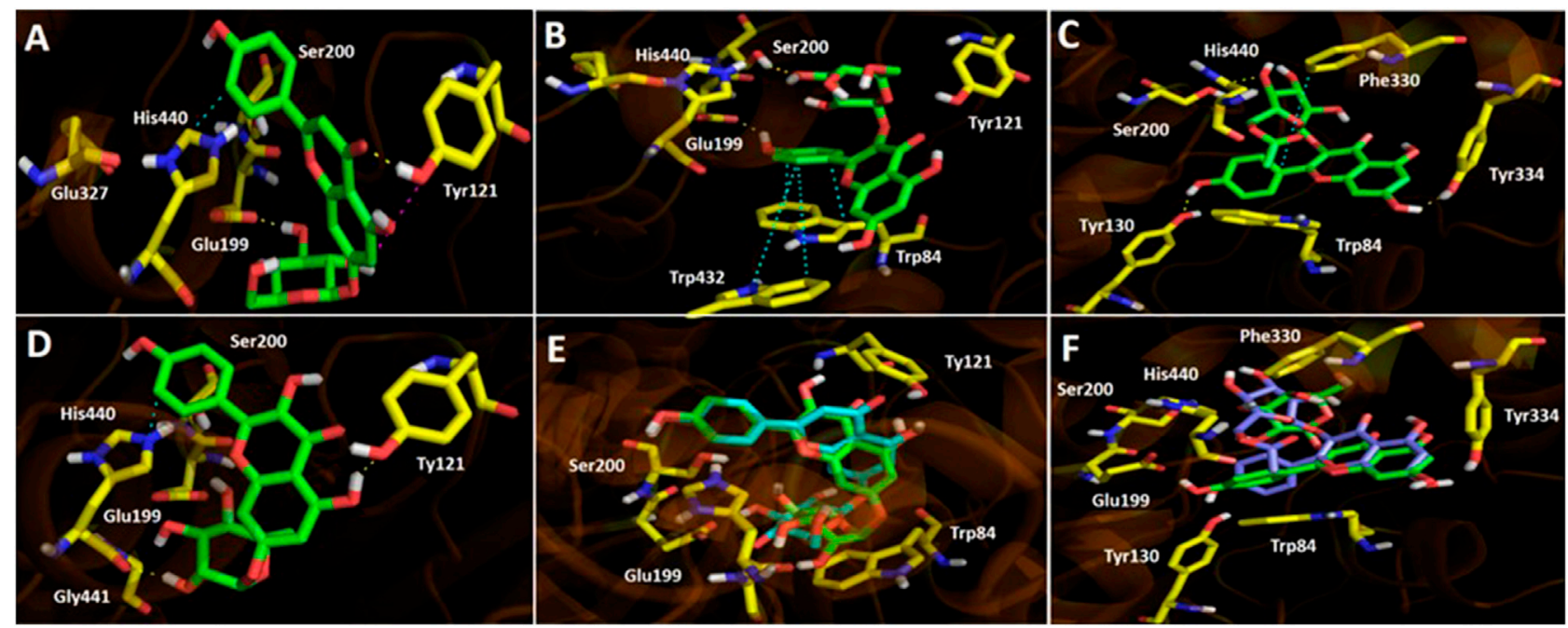

FIGURE 3 | Predicted binding mode and predicted intermolecular interactions among selected flavonoids and the residues of Torpedo californica acetylcholinesterase (TCAChE) catalytic site. (A) Apigenin 7-O-glucoside into the catalytic site (two H-bondings with Glu199 and Tyr121; T-Shaped with Tyr 121 and $\pi-\pi$ with His440). (B) Kaempferol-3-O-galactoside into the catalytic site (two H-bondings with Glu199 and Ser200; two $\pi-\pi$ interactions with Trp84 and Trp432). (C) Kaempferol-3-O-acetyl-glucoside into the catalytic site (three $\mathrm{H}$-bondings with Tyr130, Tyr334 and His440; one $\pi-\pi$ interaction with His440). (D) Kaempferol-7rhamnoside into the catalytic site (two H-bondings with Tyr121 and Gly441; one $\pi-\pi$ interaction with His440). (E) Apigenin 7-O-glucoside (green) and kaempferol-7rhamnoside (cyan) overlapped into the catalytic site. (F) Kaempferol-3-O-galactoside (green) and kaempferol-3-O-acetyl-glucoside (magenta) overlapped into the catalytic site.

hydrogen bond interactions performed by kaempferol-3$\mathrm{O}$-galactoside and kaempferol-3-O-acetyl-glucoside, as well as the $\pi-\pi$ interactions (especially compared to T-shaped interaction in apigenin-7-O-glucoside and only one $\pi-\pi$ interaction in kaempferol-7-rhamnoside) performed by the phenyl ring of the $4 H$-chromen-4-one in kaempferol-3-O-galactoside with the indole rings of $\operatorname{Trp} 84$ and $\operatorname{Trp} 432$ and with the same phenyl moiety in kaempferol-3-O-acetyl-glucoside and Phe330 of the TcAChE. The hydrogen bonding and the $\pi-\pi$ interactions mentioned above must be stronger in kaempferol-3$O$-galactoside and kaempferol-3-O-acetyl-glucoside than those for apigenin-7-O-glucoside and kaempferol-7-rhamnoside. For example, Trp bears electron-deficient aromatic rings, whereas the phenyl ring of kaempferol-3-O-galactoside possesses an electrondonating hydroxyl group $(-\mathrm{OH})$ causing a stronger interaction than apigenin-7-O-glucoside.

Docking results on $h \mathrm{BChE}$ for the four flavonoids described above, behaved similarly than in TcAChE. The binding energies of kaempferol-3-O-galactoside and kaempferol-3-O-acetyl- glucoside showed to be better $(-8.92$ and $-8.36 \mathrm{kcal} / \mathrm{mol}$ respectively) compared to apigenin-7-O-glucoside and kaempferol-7-rhamnoside $(-5.93$ and $-3.38 \mathrm{kcal} / \mathrm{mol})$, suggesting once again that the inhibitory activity over $h \mathrm{BChE}$ lies predominantly in those derivatives which bear the sugar moieties at position 3 - of the $4 \mathrm{H}$-chromen-4-one core. The binding modes of compounds into the catalytic site of $h \mathrm{BChE}$ were different this time related to results seen in TcAChE. Kaempferol-3-O-galactoside and kaempferol-3-O-acetylglucoside are completely overlapped, as well as apigenin-7$O$-glucoside and kaempferol-7-rhamnoside are arranged in the same manner with a slightly difference. This feature makes that the $4 H$-chromen-4-one moieties of the first two flavonoids to be in opposite positions related to the second ones (Figure 4). Binding energies for $h \mathrm{BChE}$ turned out to be better than for TcAChE in docking experiments, what is partially divergent with our experimental inhibition assays, where the $A$. copa infusion showed an $\mathrm{IC}_{50}=3.92 \pm 0.09 \mu \mathrm{g} / \mathrm{ml}$ for $T c A C h E$ and an $\mathrm{IC}_{50}=$ $44.13 \pm 0.10 \mu \mathrm{g} / \mathrm{ml}$ for $h \mathrm{BChE}$. 

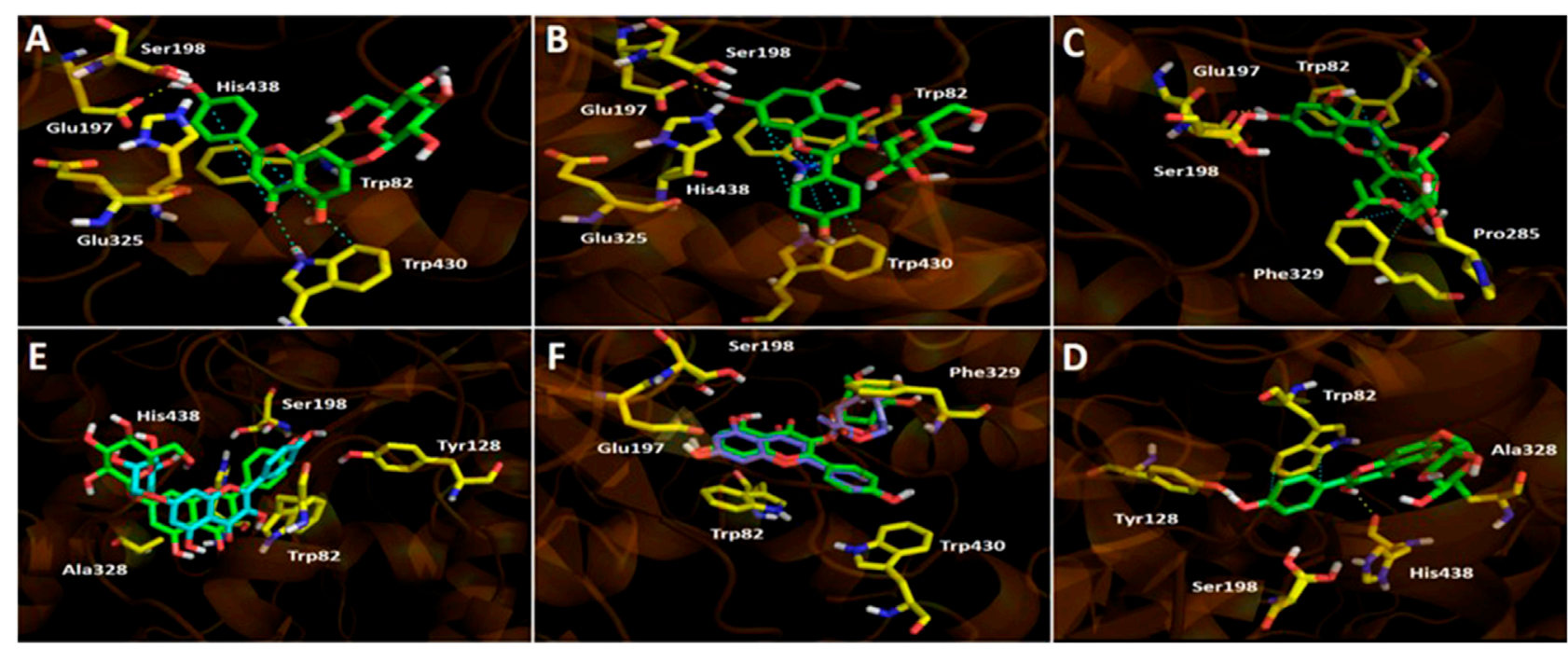

FIGURE 4 | Predicted binding mode and predicted intermolecular interactions among apigenin 7-O-glucoside, kaempferol-3-O-galactoside, kaempferol-3O-acetyl-glucoside, and kaempferol-7-rhamnoside and the residues of human butyrylcholinesterase ( $h$ BuChE) catalytic site. (A) Apigenin 7-O-glucoside into the catalytic site (one H-bond interaction with Glu197; one $\pi-\pi$ with Trp430). (B) Kaempferol-3-O-galactoside into the catalytic site (one H-bond interaction with Glu197; two $\pi-\pi$ interactions with Trp82 and Trp430). Kaempferol-3-O-acetyl-glucoside into the catalytic site (two H-bondings with Glu197 and Pro285; three $\pi-\pi$ interaction with Trp82 and Phe329). (C) Kaempferol-3-O-acetyl-glucoside into the catalytic site (two H-bondings with Glu197 and Pro285; three $\pi-\pi$ interaction with Trp82 and Phe329). (D) Kaempferol-7-rhamnoside into the catalytic site (two H-bondings with Tyr128 and His438; one $\pi-\pi$ interaction with Trp82). (E) Apigenin 7-O-glucoside (green) and kaempferol-7-rhamnoside (cyan) overlapped into the catalytic site. (F) Kaempferol-3-O-galactoside (green) and kaempferol-3-O-acetyl-glucoside (magenta) overlapped into the catalytic site.

\section{Antiprotozoal Activity Evaluation.}

To assess the potential activity from Artemisia copa infusion, we have performed a screening test against Trypanosoma cruzi and Leishmania amazonensis. Protozoan parasites are a significant cause of infectious diseases with high morbidity and mortality. Besides, Chagas and Leishmaniasis diseases have a great impact on developing countries, and no new treatments have been developed in the last 40 years (Costa et al., 2018). Artemisia copa showed trypanocidal activity against Trypanosoma cruzi with a $\mathrm{LD}_{50}$ of $131.8 \mu \mathrm{g} / \mathrm{ml}$ and against Leishmania amazonensis with a $\mathrm{LD}_{50}$ of $457 \mu \mathrm{g} / \mathrm{ml}$ (Supplementary Table S2, Supplementary Figure S3, Supplementary Material). Artemisinic acid is a sesquiterpene isolated from A. annua and a very important precursor for the production of artemisinin (Kong et al., 2013). Moreover, the antiprotozoal properties of artemisinin derivatives have been reviewed in detail (Loo et al., 2017). Asteraceae family plants play an important contribution to the development of new trypanocidal and leishmanicidal agents (Moraes Neto et al., 2019). The Artemisia genus has been pointed out as interesting antiprotozoal agents (Emami et al., 2012). The essential oils from Artemisia campestris L. and Artemisia herba-alba showed $\mathrm{IC}_{50}$ of 44 and $68 \mu \mathrm{g} / \mathrm{ml}$ against Leishmania infantum (Aloui et al., 2016). While, Artemisia annua showed $\mathrm{IC}_{50}$ of $22 \mu \mathrm{g} / \mathrm{ml}$ against Leishmania donovani (Sen et al., 2007; van der Kooy and Sullivan, 2013). The results of in vitro studies indicate that the infusion from Artemisia copa displayed low trypanocidal activity against Trypanosoma cruzi and very low activity against Leishmania amazonensis. The antiprotozoal properties of the flavonoid eupatorin isolated from the genus Stevia (Asteraceae) was investigated. Eupatorin displayed activity against Tripanozoma cruzi epimastigotes and trypomastigotes. Additionally, the activity against Leishmania braziliensis and the cytotoxicity on Vero cells were evaluated (Beer et al., 2016). The antiprotozoal properties of 7-demethylsuberosin isolated from the bark of Citropsis articulata (Willd. ex Spreng.) Swingle and M.Kellerm. (Rutaceae) was reported against Plasmodium falciparum (Lacroix et al., 2011). The antiprotozoal properties of the infusion of A. copa has not been previously reported and additional studies are necessary to explore the antiprotozoal activities.

\section{CONCLUSION}

The potential antioxidant, antiprotozoal, and cholinesterase inhibition activity plus the chemical profiling of Artemisia copa infusion were assessed. UHPLC-MS were used for the first time to detected 47 compounds in the infusion of the Altiplano plant, and to the best of our knowledge, is the first investigation on the cholinesterase inhibition activity. Our docking studies suggest that the inhibitory activity over the enzymes lies predominantly on kaempferol-3-O-galactoside and kaempferol-3-O-acetyl-glucoside. A. copa could be recommended as a supplementary beverage with cholinesterase inhibition potentials. The antiprotozoal evaluation showed no significant results against T. cruzi and L. amazonensis. However, should be 
considered that activity was evaluated in extracts where the potential active product(s) against these protozoa would be highly diluted. This study may contribute to a better understanding of the chemistry and biological activity of this plant.

\section{DATA AVAILABILITY STATEMENT}

The datasets presented in this study can be found in online repositories. The names of the repository/repositories and accession number(s) can be found in the article/Supplementary Material.

\section{AUTHOR CONTRIBUTIONS}

MS, JG, MJL-F, JE, and C F-G wrote and revised the manuscript, JR-P, MJL-F, AG, and AP analyzed the data, AP, and JP-R performed the antioxidant assays, JG and KS performed the antiprotozoal assays.

\section{REFERENCES}

Abdel-Salam, O. M. E., Youness, E. R., Mohammed, N. A., Yassen, N. N., Khadrawy, Y. A., El-Toukhy, S. E., et al. (2016). Novel neuroprotective and hepatoprotective effects of citric acid in acute malathion intoxication. Asian Pac. J. Trop. Med. 9, 1181-1194. doi:10.1016/j.apjtm.2016.11.005

Adamo, C., and Barone, V. (1999). Toward reliable density functional methods without adjustable parameters: the PBE0 model. J. Chem. Phys. 110, 6158-6170. doi: $10.1063 / 1.478522$

Aloui, Z., Messaoud, C., Haoues, M., Neffati, N., Bassoumi Jamoussi, I., EssafiBenkhadir, K., et al. (2016). Asteraceae Artemisia campestris and Artemisia herba-alba essential oils trigger apoptosis and cell cycle arrest in Leishmania infantum promastigotes. Evid. Based Complement Alternat. Med. 2016, 9147096. doi:10.1155/2016/9147096

Andriani, G., Chessler, A.-D. C., Courtemanche, G., Burleigh, B. A., and Rodriguez, A. (2011). Activity in vivo of anti-trypanosoma cruzi compounds selected from a high throughput screening. PLoS Negl. Trop. Dis. 5, e1298. doi:10.1371/ journal.pntd.0001298

Ardiles, A., Barrientos, R., Simirgiotis, M. J., Bórquez, J., Sepúlveda, B., and Areche, C. (2018). Gastroprotective activity of Parastrephia quadrangularis (Meyen) Cabrera from the Atacama Desert. Molecules 23, 2361. doi:10.3390/molecules23092361

Areche, C., Hernandez, M., Cano, T., Ticona, J., Cortes, C., Simirgiotis, M., et al. (2020). Corryocactus brevistylus (K. Schum. Ex vaupel) Britton \& Rose(Cactaceae) antioxidant, gastroprotective effects, and metabolomic profiling by ultrahigh-pressure liquid chromatography and electrospray high resolution orbitrap tandem mass spectrometry. Front. Pharmacol. 11, 417. doi:10.3389/fphar.2020.00417

Arze, J. B. L., Collin, G., Garneau, F. X., Jean, F. I., and Gagnon, H. (2004). Essential oils from Bolivia. iii. Asteraceae: Artemisia copa (Philippi). J. Essent. Oil Res. 16, 554-557. doi:10.1080/10412905.2004.9698796

Barrientos, R. E., Ahmed, S., Cortés, C., Fernández-Galleguillos, C., Romero-Parra, J., Simirgiotis, M. J., et al. (2020a). Chemical fingerprinting and biological evaluation of the endemic Chilean fruit Greigia sphacelata (Ruiz and Pav.) Regel (Bromeliaceae) by UHPLC-PDA-orbitrap-mass spectrometry. Molecules 25, 3750. doi:10.3390/molecules 25163750

Barrientos, R., Fernández-Galleguillos, C., Pastene, E., Simirgiotis, M., RomeroParra, J., Ahmed, S., et al. (2020b). Metabolomic analysis, fast isolation of phenolic compounds, and evaluation of biological activities of the bark from Weinmannia trichosperma cav. (Cunoniaceae). Front. Pharmacol. 11, 780. doi:10.3389/fphar.2020.00780

\section{FUNDING}

This research received funds from FONDECYT regular 1180059, ML-F received funds from MINEDUC-UA project code ANT 1856. CF-G acknowledges post doctorate grant 3190794 . JE gratefully acknowledges funding from CONICYT (PAI/ ACADEMIA 79160109

\section{ACKNOWLEDGMENTS}

We acknowledge Milena Rios for her help in in vitro microplate assays.

\section{SUPPLEMENTARY MATERIAL}

The Supplementary Material for this article can be found online at: https://www.frontiersin.org/articles/10.3389/fphar.2020.594174/ full\#supplementary-material

Beer, M. F., Frank, F. M., Germán Elso, O., Ernesto Bivona, A., Cerny, N., Giberti, G., et al. (2016). Trypanocidal and leishmanicidal activities of flavonoids isolated from Stevia satureiifolia var. satureiifolia. Pharm. Biol. 54, 2188-2195. doi:10.3109/13880209.2016.1150304

Benzie, I. F. F., and Strain, J. J. (1996). The ferric reducing ability of plasma (FRAP) as a measure of "antioxidant power": the FRAP assay. Anal. Biochem. 239, 70-76. doi:10.1006/abio.1996.0292

Bora, K. S., and Sharma, A. (2011). The genus Artemisia: a comprehensive review. Pharm. Biol. 49, 101-109. doi:10.3109/13880209.2010.497815

Catalán, J., Marcial, G., Schuff, C., Perotti, M., and Catalán, C. (2007). Composición química y actividad antioxidante del aceite esencial y extractos de Artemisia copa. Boletín Latinoam. y del Caribe Plantas Med. y Aromáticas 6, 238-239.

Catalan, C. A. N., Torres, O. A., and De Iglesias, D. I. A. (1982). Constituents of the essential oil from Artemisia copa philippi (compositae). Essenze Deriv. Agrum. 51, 331-335.

Coelho, M., Rocha, C., Cunha, L. M., Cardoso, L., Alves, L., Lima, R. C., et al. (2016). Influence of harvesting factors on sensory attributes and phenolic and aroma compounds composition of Cymbopogon citratus leaves infusions. Food Res. Int. 89, 1029-1037. doi:10.1016/j.foodres.2016.07.008

Costa, R. S., Souza Filho, O. P., Júnior, O. C. S. D., Silva, J. J., Hyaric, M. L., Santos, M. A. V., et al. (2018). In vitro antileishmanial and antitrypanosomal activity of compounds isolated from the roots of Zanthoxylum tingoassuiba. Rev. Bras. Farmacogn. 28, 551-558. doi:10.1016/j.bjp.2018.04.013

de la Fuente, R. J., Diaz, O. I., Morales, I., Oberti, J. C., Sosa, V. E., and Herz, W. (1986). Sesquiterpene Lactones and Flavones from Artemisia copa. Planta Med. 52, 243. doi:10.1055/s-2007-969139

De Nucci, A. M. P. (1988). La medicina tradicional del noroeste argentino: historia y presente. Ediciones Colihue S.R.L..

Echiburu-Chau, C., Pastén, L., Parra, C., Bórquez, J., Mocan, A., and Simirgiotis, M. J. (2017). High resolution UHPLC-MS characterization and isolation of main compounds from the antioxidant medicinal plant Parastrephia lucida (Meyen). Saudi Pharm. J. 25, 1032-1039. doi:10.1016/j.jsps.2017.03.001

Ellman, G. L., Courtney, K. D., Andres, V., and Featherstone, R. M. (1961). A new and rapid colorimetric determination of acetylcholinesterase activity. Biochem. Pharmacol. 7, 88-95. doi:10.1016/0006-2952(61)90145-9

Emami, S. A., Zamanai Taghizadeh Rabe, S., Ahi, A., and Mahmoudi, M. (2012). Inhibitory activity of eleven Artemisia species from Iran against Leishmania major parasites. Iran J. Basic Med. Sci. 15, 807-811.

Giberti, G. C. (1983). Herbal folk medicine in northwestern Argentina: Compositae. J. Ethnopharmacol. 7, 321-341. doi:10.1016/0378-8741(83)90006-5 
Gorzalczany, S., Moscatelli, V., Acevedo, C., and Ferraro, G. (2013a). Spasmolytic activity of Artemisia copa aqueous extract and isolated compounds. Nat. Prod. Res. 27, 1007-1011. doi:10.1080/14786419.2012.688049

Gorzalczany, S., Moscatelli, V., and Ferraro, G. (2013b). Artemisia copa aqueous extract as vasorelaxant and hypotensive agent. J. Ethnopharmacol. 148, 56-61. doi:10.1016/j.jep.2013.03.061

Gutiérrez, B., Muñoz, C., Osorio, L., Fehér, K., Illyés, T.-Z., Papp, Z., et al. (2013). Aromatic glycosyl disulfide derivatives: evaluation of their inhibitory activities against Trypanosoma cruzi. Bioorg. Med. Chem. Lett. 23, 3576-3579. doi:10. 1016/j.bmcl.2013.04.030

Huang, D., Ou, B., Hampsch-Woodill, M., Flanagan, J. A., and Prior, R. L. (2002). High-throughput assay of oxygen radical absorbance capacity (ORAC) using a multichannel liquid handling system coupled with a microplate fluorescence reader in 96-well format. J. Agric. Food Chem. 50, 4437-4444. doi:10.1021/ jf0201529

Hung, T. M., Lee, J. S., Chuong, N. N., Kim, J. A., Oh, S. H., Woo, M. H., et al. (2015). Kinetics and molecular docking studies of cholinesterase inhibitors derived from water layer of Lycopodiella cernua (L.) Pic. Serm. (II). Chem. Biol. Interact. 240, 74-82. doi:10.1016/j.cbi.2015.07.008

Jafari, N., Zargar, S. J., Delnavazi, M.-R., and Yassa, N. (2017). Cell cycle arrest and apoptosis induction of phloroacetophenone glycosides and caffeoylquinic acid derivatives in gastric adenocarcinoma (AGS) cells. Anticancer. Agents Med. Chem. 18, 610-616. doi:10.2174/ 1871520618666171219121449

Jaiswal, P., Kumar, P., Singh, V. K., and Singh, D. K. (2010). Enzyme inhibition by molluscicidal components of Myristica fragrans Houtt. In the nervous Tissue of snail Lymnaea acuminata. Enzyme Res. 2010, 478746. doi:10.4061/2010/ 478746

Jeong, S. Y., Sang, H. S., and Young, C. K. (2008). Neuroprotective limonoids of root bark of Dictamnus dasycarpus. J. Nat. Prod. 71, 208-211. doi:10.1021/ np070588o

Kaneda, M. (1990). Lilioside a from Lilium longiflorum: synthesis and absolute configuration. Phytochemistry 29, 3559-3564. doi:10.1016/0031-9422(90) 85276-L

Kang, S. Y., Lee, K. Y., Sung, S. H., Park, M. J., and Kim, Y. C. (2001). Coumarins isolated from Angelica gigas inhibit acetylcholinesterase: structure-activity relationships. J. Nat. Prod. 64, 683-685. doi:10.1021/np000441w

Khan, H., MaryaAmin, S., Kamal, M. A., and Patel, S. (2018). Flavonoids as acetylcholinesterase inhibitors: current therapeutic standing and future prospects. Biomed. Pharmacother. 101, 860-870. doi:10.1016/j.biopha.2018. 03.007

Khan, M. T. H., Orhan, I., Şenol, F. S., Kartal, M., Şener, B., Dvorská, M., et al. (2009). Cholinesterase inhibitory activities of some flavonoid derivatives and chosen xanthone and their molecular docking studies. Chem. Biol. Interact. 181, 383-389. doi:10.1016/j.cbi.2009.06.024

Kong, J., Yang, Y., Wang, W., Cheng, K., and Zhu, P. (2013). Artemisinic acid: A promising molecule potentially suitable for the semi-synthesis of artemisinin. RSC Adv. 3, 76227641. doi:10.1039/c3ra40525g

Kwon, S. H., Lee, H. K., Kim, J. A., Hong, S. I., Kim, H. C., Jo, T. H., et al. (2010). Neuroprotective effects of chlorogenic acid on scopolamine-induced amnesia via anti-acetylcholinesterase and anti-oxidative activities in mice. Eur. J. Pharmacol. 649, 210-217. doi:10.1016/j.jphar.2010.09.001

Kwon, Y., Kim, H. P., Kim, M. J., and Chun, W. (2017). Acetylcholinesterase inhibitors from Angelica polymorpha stem. Nat. Prod. Sci. 23, 97-102. doi:10. 20307/nps.2017.23.2.97

Lacroix, D., Prado, S., Kamoga, D., Kasenene, J., and Bodo, B. (2011). Structure and in vitro antiparasitic activity of constituents of Citropsis articulata root bark. J. Nat. Prod. 74, 2286-2289. doi:10.1021/np2004825

Larrazabal, M. J., Palma, J., Paredes, A., Morales, G., and Mercado, A. (2018). Effect of brewing conditions on pigments and total polyphenols content and biological activities of the Acantholippia deserticola (Phil.) infusion. CyTA J. Food 16, 588-595. doi:10.1080/19476337.2018.1441189

Larrazabal-Fuentes, M., Palma, J., Paredes, A., Mercado, A., Neira, I., Lizama, C., et al. (2019). Chemical composition, antioxidant capacity, toxicity and antibacterial activity of the essential oils from Acantholippia deserticola (Phil.) Moldenke (Rica rica) and Artemisia copa Phil. (Copa copa) extracted by microwave-assisted hydrodistillation. Ind. Crops Prod. 142, 111830. doi:10. 1016/j.indcrop.2019.111830
Liu, W., Yin, D., Zhang, T., Qiao, Q., Yang, Y., and Wang, W. (2020). Phytochemical profiles and antioxidant activity of Rehmannia glutinosa from different production locations. Chem. Biodivers. 17, e2000341. doi:10. $1002 / \mathrm{cbdv} .202000341$

Loo, C. S. N., Lam, N. S. K., Yu, D., Su, X. zhuan., and Lu, F. (2017). Artemisinin and its derivatives in treating protozoan infections beyond malaria. Pharmacol. Res. 117, 192217. doi:10.1016/j.phrs.2016.11.012

Loyola, L. A., Bórquez, J., Morales, G., Araya, J., González, J., Neira, I., et al. (2001). Diterpenoids from Azorella yareta and their trichomonicidal activities. Phytochemistry 56, 177-180. doi:10.1016/S0031-9422(00)00380-0

Miño, J., Moscatelli, V., Hnatyszyn, O., Gorzalczany, S., Acevedo, C., and Ferraro, G. (2004). Antinociceptive and antiinflammatory activities of Artemisia copa extracts. Pharmacol. Res. 50, 59-63. doi:10.1016/j.phrs.2003.12.016

Montes, M., and Wilkomirsky, T. (1985). Medicina tradicional chilena. Chile: Editorial de la Universidad de Conception Chile.

Moon, J. K., and Shibamoto, T. (2009). Antioxidant assays for plant and food components. J. Agric. Food Chem. 57, 1655-1666. doi:10.1021/jf803537k

Moraes Neto, R. N., Setúbal, R. F. B., Higino, T. M. M., Brelaz-de-Castro, M. C. A., da Silva, L. C. N., and Aliança, A. S. (2019). Asteraceae plants as sources of compounds against Leishmaniasis and Chagas disease. Front. Pharmacol. 10, 477. doi:10.3389/fphar.2019.00477

Morales, G., and Paredes, A. (2014). Antioxidant activities of Lampaya medicinalis extracts and their main chemical constituents. BMC Complement. Altern. Med. 14, 1-12. doi:10.1186/1472-6882-14-259

Morales, G., Sierra, P., Mancilla, A., Paredes, A., Loyola, L. A., Gallardo, O., et al. (2003). Secondary metabolites from four medicinal plants from northern Chile: antimicrobial activity and biotoxicity against Artemia salina. J. Chil. Chem. Soc. $48,13-18$.

Moscatelli, V., Hnatyszyn, O., Acevedo, C., Megías, J., Alcaraz, M., and Ferraro, G. (2006). Flavonoids from Artemisia copa with anti-inflammatory activity. Planta Med. 72, 72-74. doi:10.1055/s-2005-873177

Mousa, A. A., El-Gansh, H. A. I., Eldaim, M. A. A., Mohamed, M. A. E. G., Morsi, A. H., and El Sabagh, H. S. (2019). Protective effect of Moringa oleifera leaves ethanolic extract against thioacetamide-induced hepatotoxicity in rats via modulation of cellular antioxidant, apoptotic and inflammatory markers. Environ. Sci. Pollut. Res. 26, 32488-32504. doi:10.1007/s11356-019-06368-4

$\mathrm{Naß}$, J., and Efferth, T. (2018). The activity of Artemisia spp. and their constituents against Trypanosomiasis. Phytomed. 47, 184-191. doi:10.1016/j.phymed.2018. 06.002

Oboh, G., Agunloye, O. M., Akinyemi, A. J., Ademiluyi, A. O., and Adefegha, S. A. (2013). Comparative study on the inhibitory effect of caffeic and chlorogenic acids on key enzymes linked to Alzheimer's disease and some pro-oxidant induced oxidative stress in rats' brain-in vitro. Neurochem. Res. 38, 413-419. doi:10.1007/s11064-012-0935-6

Oliveira, C., Cagide, F., Teixeira, J., Amorim, R., Sequeira, L., Mesiti, F., et al. (2018). Hydroxybenzoic acid derivatives as dual-target ligands: mitochondriotropic antioxidants and cholinesterase inhibitors. Front. Chem. 6, 126. doi:10.3389/fchem.2018.00126

Orhan, I. E., Belhattab, R., Şenol, F. S., Gülpinar, A. R., Hoșbaş, S., and Kartal, M. (2010). Profiling of cholinesterase inhibitory and antioxidant activities of Artemisia absinthium, A. herba-alba, A. fragrans, Marrubium vulgare, $M$. astranicum, Origanum vulgare subsp. glandulossum and essential oil analysis of two Artemisia species. Ind. Crops Prod. 32, 566-571. doi:10.1016/j.indcrop. 2010.07.005

Pérez-García, F., Marín, E., Adzet, T., and Cañigueral, S. (2001). Activity of plant extracts on the respiratory burst and the stress protein synthesis. Phytomedicine 8, 31-38. doi:10.1078/0944-7113-00018

Pan, S.-Y., Zhou, S.-F., Gao, S.-H., Yu, Z.-L., Zhang, S.-F., Tang, M.-K., et al. (2013). New perspectives on how to discover drugs from herbal medicines: CAM's outstanding contribution to modern therapeutics. Evidence-Based Complement. Altern. Med. 2013, 627375. doi:10.1155/2013/627375

Paniagua-Zambrana, N. Y., Bussmann, R. W., Echeverría, J., and Romero, C. (2020). "Artemisia absinthium L. Artemisia annua L. Artemisia copa Phil. Asteraceae," in Ethnobotany of the Andes. Editors N. Y. Paniagua-Zambrana and R. W. Bussmann (Cham, Switzerland: Springer International Publishing), 247-257

Ramirez, J. E., Zambrano, R., Sepúlveda, B., Kennelly, E. J., and Simirgiotis, M. J. (2015). Anthocyanins and antioxidant capacities of six Chilean berries by 
HPLC-HR-ESI-ToF-MS. Food Chem. 176, 106-114. doi:10.1016/j.foodchem. 2014.12.039

Rodríguez, K., Ah-Hen, K., Vega-Gálvez, A., López, J., Quispe-Fuentes, I., LemusMondaca, R., et al. (2014). Changes in bioactive compounds and antioxidant activity during convective drying of murta ( Ugni molinae T.) berries. Int. J. Food Sci. Technol. 49, 990-1000. doi:10.1111/ijfs.12392

Rodriguez, S., Pertino, M. W., Arcos, C., Reichert, L., Echeverria, J., Simirgiotis, M., et al. (2020). Isolation, gastroprotective effects and untargeted metabolomics analysis of lycium minutifolium J. Remy (solanaceae). Foods 9, 565. doi:10.3390/foods9050565

Ślusarczyk, S., Senol Deniz, F. S., Woźniak, D., Pecio, Ł., Pérez-Sánchez, H., CerónCarrasco, J. P., et al. (2019). Selective in vitro and in silico cholinesterase inhibitory activity of isoflavones and stilbenes from Belamcandae chinensis rhizoma. Phytochem. Lett. 30, 261-272. doi:10.1016/j.phytol.2019.02.006

San Francisco, J., Barría, I., Gutiérrez, B., Neira, I., Muñoz, C., Sagua, H., et al. (2017). Decreased cruzipain and gp $85 /$ trans-sialidase family protein expression contributes to loss of Trypanosoma cruzi trypomastigote virulence. Microbes Infect. 19, 55-61. doi:10.1016/j.micinf.2016.08.003

Sauter, I. P., Madrid, K. G., de Assis, J. B., Sá-Nunes, A., Torrecilhas, A. C., Staquicini, D. I., et al. (2019). TLR9/MyD88/TRIF signaling activates host immune inhibitory CD200 in Leishmania infection. JCI Insight 4 (10), e126207. doi:10.1172/jci.insight.126207

Sen, R., Bandyopadhyay, S., Dutta, A., Mandal, G., Ganguly, S., Saha, P., et al. (2007). Artemisinin triggers induction of cell-cycle arrest and apoptosis in Leishmania donovani promastigotes. J. Med. Microbiol. 56, 1213-1218. doi:10.1099/jmm.0.47364-0

Sevindik, H. G., Güvenalp, Z., Yerdelen, K. Ö., Yuca, H., and Demirezer, L. Ö. (2015). The discovery of potential anticholinesterase compounds from Achillea millefolium L. Ind. Crops Prod. 76, 873-879. doi:10.1016/j. indcrop.2015.05.088

Simirgiotis, J. M., Quispe, C., Bórquez, J., Areche, C., and Sepúlveda, B. (2016a). Fast detection of phenolic compounds in extracts of easter pears (pyrus communis) from the Atacama Desert by ultrahigh-performance liquid chromatography and mass spectrometry (UHPLC-Q/Orbitrap/MS/MS). Molecules 21, 92. doi:10.3390/molecules21010092

Simirgiotis, M. J., Quispe, C., Bórquez, J., Mocan, A., and Sepúlveda, B. (2016b). High resolution metabolite fingerprinting of the resin of Baccharis tola Phil. from the Atacama Desert and its antioxidant capacities. Ind. Crops Prod. 94, 368-375. doi:10.1016/J.INDCROP.2016. 08.037

Simirgiotis, M. J., Quispe, C., Bórquez, J., Schmeda-Hirschmann, G., Avendaño, M., Sepúlveda, B., et al. (2016c). Fast high resolution Orbitrap MS fingerprinting of the resin of Heliotropium taltalense Phil. from the Atacama Desert. Ind. Crops Prod. 85, 159-166. doi:10.1016/j.indcrop.2016. 02.054
Simirgiotis, M. J., Ramirez, J. E., Schmeda Hirschmann, G., and Kennelly, E. J (2013). Bioactive coumarins and HPLC-PDA-ESI-ToF-MS metabolic profiling of edible queule fruits (Gomortega keule), an endangered endemic Chilean species. Food Res. Int. 54, 532-543. doi:10.1016/j. foodres.2013.07.022

Szwajgier, D., and Borowiec, K. (2012). Phenolic acids from malt are efficient acetylcholinesterase and butyrylcholinesterase inhibitors. J. Inst. Brew. 118, 40-48. doi:10.1002/jib.5

Taşkın, D., Taşkın, T., and Rayaman, E. (2018). Phenolic composition and biological properties of Achillea nobilis L. subsp. neilreichii (Kerner) Formanek. Ind. Crops Prod. 111, 555-562. doi:10.1016/j.indcrop.2017.11.022

Topal, M., Gocer, H., Topal, F., Kalin, P., Köse, L. P., Gulçin, I., et al. (2016). Antioxidant, antiradical, and anticholinergic properties of cynarin purified from the Illyrian thistle (Onopordum illyricum L.). J. Enzyme Inhib. Med. Chem. 31, 266-275. doi:10.3109/14756366.2015.1018244

van der Kooy, F., and Sullivan, S. E. (2013). The complexity of medicinal plants: the traditional Artemisia annua formulation, current status and future perspectives. J. Ethnopharmacol. 150, 1-13. doi:10.1016/j.jep.2013.08.021

Zengin, G., Mollica, A., Aktumsek, A., Marie Nancy Picot, C., and Fawzi Mahomoodally, M. (2017). In vitro and in silico insights of Cupressus sempervirens, Artemisia absinthium and Lippia triphylla: bridging traditional knowledge and scientific validation. Eur. J. Integr. Med. 12, 135-141. doi:10. 1016/j.eujim.2017.05.010

Zeraik, M. L., Queiroz, E. F., Marcourt, L., Ciclet, O., Castro-Gamboa, I., Silva, D. H. S., et al. (2016). Antioxidants, quinone reductase inducers and acetylcholinesterase inhibitors from Spondias tuberosa fruits. J. Funct. Foods 21, 396-405. doi:10.1016/j.jff.2015.12.009

Zhang, L., Huang, D., Kondo, M., Fan, E., Ji, H., Kou, Y., et al. (2009). Novel highthroughput assay for antioxidant capacity against superoxide anion. J. Agric. Food Chem. 57, 2661-2667. doi:10.1021/jf8033368

Conflict of Interest: The authors declare that the research was conducted in the absence of any commercial or financial relationships that could be construed as a potential conflict of interest.

Copyright (c) 2020 Larrazábal-Fuentes, Fernández-Galleguillos, Palma-Ramírez, Romero-Parra, Sepúlveda, Galetovic, González, Paredes, Bórquez, Simirgiotis and Echeverria. This is an open-access article distributed under the terms of the Creative Commons Attribution License (CC BY). The use, distribution or reproduction in other forums is permitted, provided the original author(s) and the copyright owner(s) are credited and that the original publication in this journal is cited, in accordance with accepted academic practice. No use, distribution or reproduction is permitted which does not comply with these terms. 